OPEN ACCESS

Edited by:

Teodorico Castro Ramalho,

Universidade Federal de Lavras, Brazil

Reviewed by:

Hyun Lee,

University of Illinois at Chicago,

United States

Salvatore Guccione,

University of Catania, Italy

Daiana Mancini,

Universidade Federal de Lavras, Brazil

*Correspondence:

Nicholas Furnham

nick.furnham@/shtm.ac.uk

Carolina Horta Andrade

carolina@ufg.br

Floriano Paes Silva Jr.

floriano@ioc.fiocruz.br

tThese authors have contributed equally to this work

Specialty section:

This article was submitted to

Medicinal and Pharmaceutical

Chemistry,

a section of the journal

Frontiers in Chemistry

Received: 25 October 2019

Accepted: 30 January 2020

Published: 18 February 2020

Citation:

de Souza Neto LR, Moreira-Filho JT,

Neves BJ, Maidana RLBR, Guimarães

$A C R$, Furnham N, Andrade $\mathrm{CH}$ and

Silva FP Jr (2020) In silico Strategies

to Support Fragment-to-Lead

Optimization in Drug Discovery.

Front. Chem. 8:93.

doi: 10.3389/fchem.2020.00093

\section{In silico Strategies to Support Fragment-to-Lead Optimization in Drug Discovery}

\author{
Lauro Ribeiro de Souza Neto ${ }^{1 \dagger}$, José Teófilo Moreira-Filho ${ }^{2 t}$, Bruno Junior Neves ${ }^{2,3 t}$, \\ Rocío Lucía Beatriz Riveros Maidana ${ }^{1,4}$, Ana Carolina Ramos Guimarães ${ }^{4}$, \\ Nicholas Furnham ${ }^{5 *}$, Carolina Horta Andrade ${ }^{2 *}$ and Floriano Paes Silva Jr. ${ }^{1 *}$ \\ ${ }^{1}$ LaBECFar - Laboratório de Bioquímica Experimental e Computacional de Fármacos, Instituto Oswaldo Cruz, Fundação \\ Oswaldo Cruz, Rio de Janeiro, Brazil, ${ }^{2}$ LabMol - Laboratory for Molecular Modeling and Drug Design, Faculdade de \\ Farmácia, Universidade Federal de Goiás, Goiânia, Brazil, ${ }^{3}$ Laboratory of Cheminformatics, Centro Universitário de Anápolis \\ - UniEVANGÉLICA, Anápolis, Brazil, ${ }^{4}$ Laboratório de Genômica Funcional e Bioinformática, Instituto Oswaldo Cruz, \\ Fundação Oswaldo Cruz, Rio de Janeiro, Brazil, ${ }^{5}$ Department of Infection Biology, London School of Hygiene and Tropical \\ Medicine, London, United Kingdom
}

Fragment-based drug (or lead) discovery (FBDD or FBLD) has developed in the last two decades to become a successful key technology in the pharmaceutical industry for early stage drug discovery and development. The FBDD strategy consists of screening low molecular weight compounds against macromolecular targets (usually proteins) of clinical relevance. These small molecular fragments can bind at one or more sites on the target and act as starting points for the development of lead compounds. In developing the fragments attractive features that can translate into compounds with favorable physical, pharmacokinetics and toxicity (ADMET-absorption, distribution, metabolism, excretion, and toxicity) properties can be integrated. Structure-enabled fragment screening campaigns use a combination of screening by a range of biophysical techniques, such as differential scanning fluorimetry, surface plasmon resonance, and thermophoresis, followed by structural characterization of fragment binding using NMR or X-ray crystallography. Structural characterization is also used in subsequent analysis for growing fragments of selected screening hits. The latest iteration of the FBDD workflow employs a high-throughput methodology of massively parallel screening by X-ray crystallography of individually soaked fragments. In this review we will outline the FBDD strategies and explore a variety of in silico approaches to support the follow-up fragment-to-lead optimization of either: growing, linking, and merging. These fragment expansion strategies include hot spot analysis, druggability prediction, SAR (structure-activity relationships) by catalog methods, application of machine learning/deep learning models for virtual screening and several de novo design methods for proposing synthesizable new compounds. Finally, we will highlight recent case studies in fragment-based drug discovery where in silico methods have successfully contributed to the development of lead compounds.

Keywords: fragment-based, drug discovery, lead discovery, in silico methods, machine learning, de novo design, optimization, hot spot analysis 


\section{INTRODUCTION}

\section{Fragment-Based Drug Discovery}

Since the inception of fragment-based drug discovery (FBDD) over 20 years ago it has become an established technology used in both industry and academia (Hubbard, 2015). FBDD offers an attractive approach for effectively exploring the chemical space for binding a target protein. In conventional high-throughput screening (HTS) campaigns, large libraries of often complex compounds are screened for activity against a target (Hall et al., 2014). In contrast, FBDD use relatively small libraries of low complexity compounds representing fragments of larger more drug-like compounds. By reducing the complexity of the chemicals screened more of the potential binding sites of a target protein can be explored through the binding promiscuity of the fragments (Thomas et al., 2017). Where fragments do bind, albeit with lower potency than the drug-like molecules of HTS, they offer good starting points to design larger higher affinity binders using knowledge of the protein structure as a template to generate compounds with greater ligand efficiency (improved per atom binding energy to the target). This bottom-up approach means that a greater range of chemical space can be explored, leading quickly to higher affinity lead compounds with greater specificity (Patel et al., 2014).

FBDD projects require relatively lower investments in research and development (R\&D) than HTS (Davis and Roughley, 2017). An example is the discovery of vemurafenib (Zelboraf $^{\mathrm{TM}}$ ), the first fragment-derived drug, which moved relatively very quickly (6 years) between the phases of $R \& D$ pipeline before reaching Food and Drug Association (FDA) approval (Erlanson et al., 2016). Thus, FBDD provides attractive opportunities for the drug discovery field.

\section{Output of Structure-Enabled Fragment Screening Campaigns}

FBDD workflows are multi-step starting with target selection and protein isolation and followed by an initial screen of the fragment library using biophysical techniques such as nuclear magnetic resonance (NMR), surface plasmon resonance (SPR), thermal-shift assay, microscale thermophoresis (MST), mass spectrometry, and others. For fragments which show evidence of binding, a further step of hit validation and characterization occurs principally using X-ray crystallography (Verdonk and Hartshorn, 2004). Using hit characterization, an iterative cycle of fragment development can occur employing a range of in silico and experimental techniques. Advances in this protocol try to compress the process by combining the initial fragment screen with the hit characterization. This has been implemented in a high throughput FBDD platform called XChem located at the United Kingdom's national synchrotron the Diamond Light Source (Cox et al., 2016). It uses the ability to produce and handle a large number of crystals of the target protein to screen the fragment library by soaking each individual crystal with a fragment and then using X-ray crystallography to determine which fragments have bound and where. Though this high throughput technique often provides multiple hits, care needs to be taken in interpreting the significance of the hit.
Promiscuous fragments may bind parts of the protein which are not involved in the protein function and therefore are unlikely to yield a successful inhibitor. Additionally, as the fragments are by their very nature weak binders and X-ray crystallography being a sensitive technique, observed binding events might be transient and not easily reproducible. It is therefore important to confirm hits with orthogonal structural (e.g., NMR), biophysical techniques (SPR, MST, etc.) or in vitro biological assays.

\section{Fragment Libraries}

A crucial step in FBDD process is in the development and choice of the fragment library used in the screening campaign. Several fragment libraries have been developed that exploit certain properties or chemistries. An example of a fragment library is the Diamond-SGC Poised Library (DSPL) (Cox et al., 2016). This has been developed for use with high-throughput XChem platform and consists of around 760 fragments that have been selected to contain at least one functional group that is open to rapid, cheap follow-up synthesis using robust well-characterized reactions (poised) and maximizing chemical diversity. Other fragment libraries optimize other properties such as solubility, 3D traits or based on subsets of existing drugs and related molecules such as natural products (Schuffenhauer et al., 2005). The fragment libraries generally share similar properties of "Rule of 3" compliant i.e., less than $300 \mathrm{Da}$ molecular weight, 3 or less hydrogen bond donors, 3 or less hydrogen bond acceptors and $C \log P$ no more than 3. In addition, they are soluble in dimethyl sulfoxide (DMSO) or phosphate buffered saline. Fragment libraries generally tend to be $<1,000$ fragments, which is significantly less than the many millions of compounds screened in high-throughput and high content screening campaigns (Trevizani et al., 2017).

\section{Fragment Expansion Strategies}

Once the fragment screen has been completed and hits characterized, the next step is the challenge of expanding these fragments to generate larger molecular entities with high binding affinity and demonstrating inhibition activity. There are several strategies that can be followed (Lamoree and Hubbard, 2017) (Figure 1). One option is to use expert medicinal chemistry advice to design and synthesize larger molecules based on the protein and the fragment pose. Another approach is to define vectors along the fragment molecule based on the steric hindrance of the protein target in which the fragment can be expanded. The fragment is then searched for within a large library of synthesizable (or purchasable) molecules which are bigger by between one and three heavy atoms along the identified vectors. These expanded fragments can be synthesized and soaked/cocrystallized and re-screened by X-ray crystallography. Expanded fragments that show improved binding can be further extended or structurally modified using the same process, with this cycle continuing until a larger high-affinity binding entity is reached.

An alternative to this "small steps" approach is to try to get a larger higher affinity binding molecule in a single step. This can be achieved by having an in silico method using the fragment in a substructure search of a large purchasable compound library (e.g., Zinc15), and to virtually screen the results using the pose 


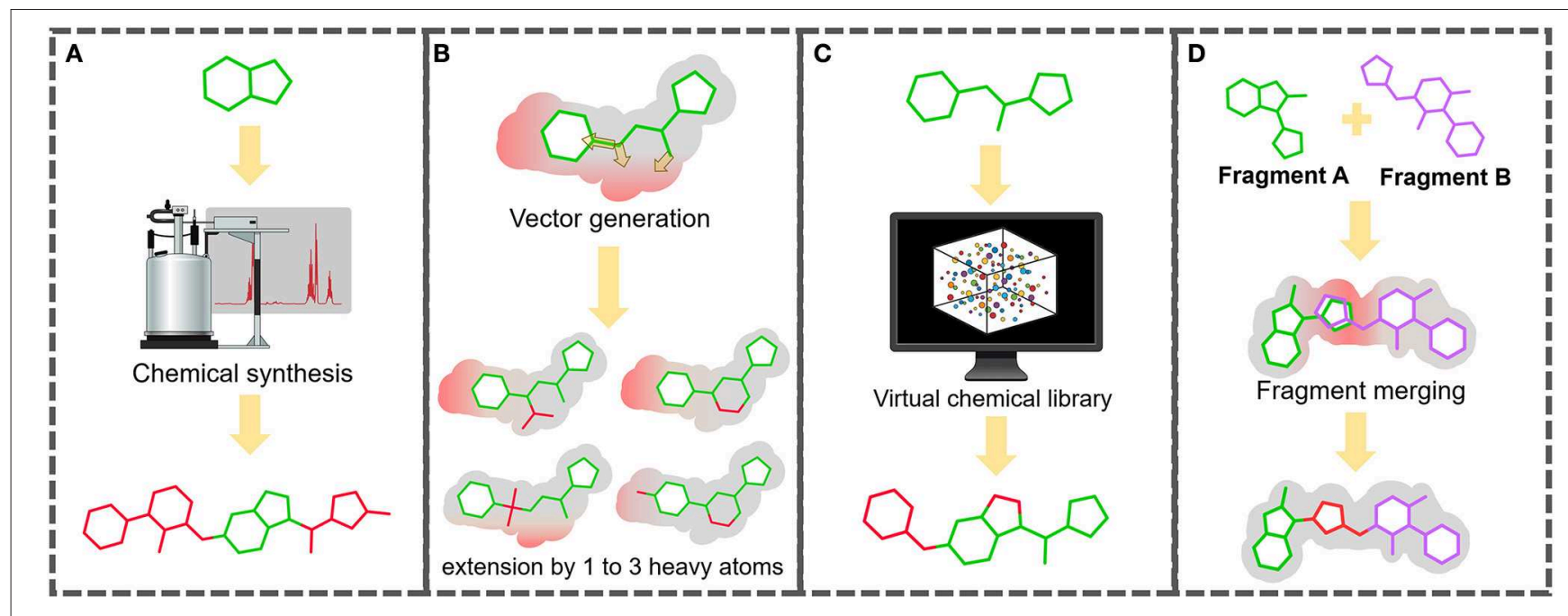

FIGURE 1 | Multiple routes to expanding fragment to more drug-like molecule with improved binding affinity. (A) Traditional medicinal chemistry route: knowledge-based design and synthesis. (B) "Small steps" route: successive cycles of extension of the fragment hit by 1-3 heavy atoms through vectors defined by high-resolution structural characterization methods, such as X-ray crystallography. (C) "Large leaps" or "SAR by catalog" route: from fragment to rule of 5 compliant molecules using virtual screening of commercial compound libraries. (D) Fragment merging route: bridging two overlapping fragments bound at neighbor sites. Regardless of the route, expanded fragments should be checked for biological activity using in vitro, ex vivo, or in vivo assays.

of the fragment to dock the molecules and rank them based on docking parameters (Trevizani et al., 2017). The top-ranking virtual screening hits can then be co-crystallized and well as evaluated in vitro and in vivo. A final expansion option is to link or merge fragments that hit near to each other or within the same site (Davis and Roughley, 2017). The combined fragments can then be further expanded using the approaches described previously. It is vital that as expansion progresses in vitro and in vivo assays are conducted to asses activity of the new molecules.

In the next section, we will discuss in depth the main optimization approaches used for a fragment structurally characterized in a binding site of its target. Further sections will describe existing software tools or modeling techniques (e.g., machine learning) employed for taking a fragment hit thorough the path for becoming a lead compound-a process known as fragment-to-lead (F2L) - for drug development and conclude by presenting case studies where in silico strategies have been successfully utilized to support the F2L optimization process.

\section{FRAGMENT OPTIMIZATION APPROACHES}

After the hit identification in a FBDD campaign, the fragment moves forward to the optimization phase. This optimization takes into account the structural characteristics of the ligand as well as its binding site. The principle in using fragments relies on the premise that these molecular entities are more efficient ligands compared to drug-like molecules, and their structures can be further optimized more efficiently. In fact, this constitutes one of its many advantages. As small entities, molecular fragments can be iteratively optimized to show a better pharmacokinetic profile in the later development stages. Druglike molecules may contain functional groups that contribute poorly to protein binding or, in some cases, can even disrupt the protein-ligand interaction. On the other hand, fragments often form high-quality interactions able to more easily bind to the protein target, translating to a greater number of hits. Figure 2 depicts schematically this concept.

Another advantage of FBDD is the potential for faster hit progression through the campaign, since the fragments are usually structurally simple and many follow-up compounds can be easily purchased from commercial databases (e.g., MolPort, ZINC15, and ChemBridge) instead of being synthesized. Another important characteristic often used to defend this approach is the high hit rates. In this sense, high hit rates means that the FBDD yields relatively more hits in comparison to the traditional methods such as HTS (Coutard et al., 2014; Mondal et al., 2015). This is due the inversely related nature between molecular complexity and the binding probability (Hann et al., 2001). Other advantages includes the more efficient chemical space sampling (Coutard et al., 2014; Mondal et al., 2015) and the relative low cost to implement the FBDD, as it can be seen from comparing the usual size of the HTS library (thousands of compounds) with fragment libraries (hundreds of compounds) (Macarron et al., 2011).

Assessment of the interactions between the fragment and its binding site should be carefully performed for further identification of synthetically accessible vectors on the ligand. Although $\mathrm{x}$-ray crystallography data is a valuable technique in fragment optimization, is important to keep in mind that the observed structural data only represents a snapshot of the system under investigation. It's been known that the ligand affinity can be affected by the structural protein dynamics without changing the ligand-binding interface (Matias et al., 2000; Seo et al., 2014). This complex dynamic environment (Henzler-Wildman and Kern, 2007; Boehr et al., 2009) can affect small and weak ligands as 


\section{A}

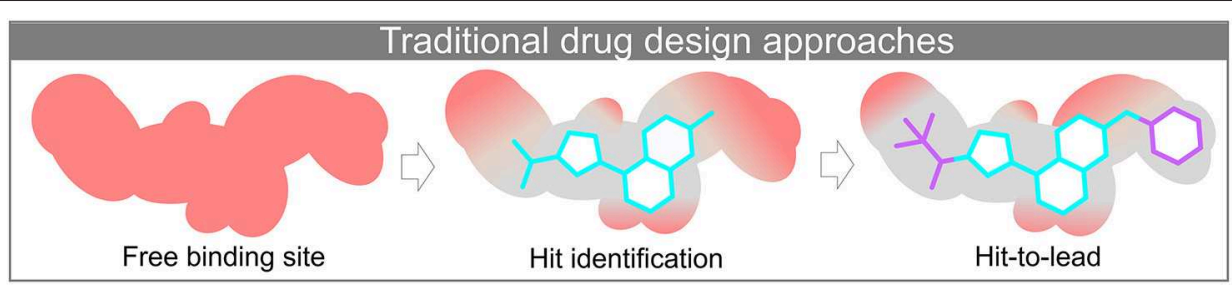

B

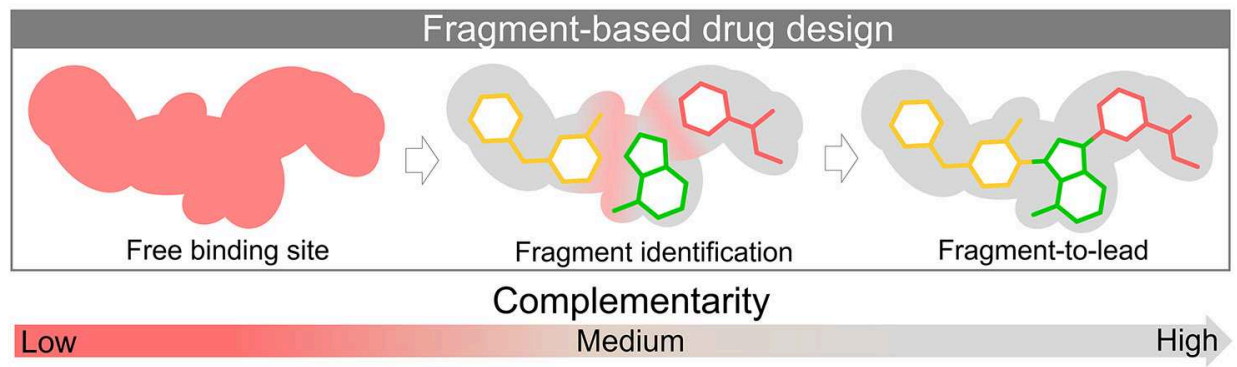

FIGURE 2 | Discovery and structural-optimization of drug-like molecules (A) and fragments (B) using protein target information. The surface represents the binding site. The red and gray colors represent the level of complementarity of ligand with the active site. Pockets with low complementarity with ligand are colored in red; pockets with high complementarity with ligand are highlighted in gray.

fragments. With this in mind, many methods can additionally be used to guide the fragment identification/optimization either providing complementary data (e.g., thermodynamic data) or acting as orthogonal approaches (Ciulli, 2013a). These methods are mostly biophysical (Shuker et al., 1996; Lo et al., 2004; Navratilova and Hopkins, 2010; Pedro and Quinn, 2016) and their use has some advantages such as, direct measurement of the binding, detection of small ligands with low affinity, and not needing any prior information about the protein function (Ciulli, 2013b). Despite the supremacy of biophysical methods, biochemical approaches are increasingly being used (Godemann et al., 2009; Boettcher et al., 2010; Mondal et al., 2015) in FBLD.

In addition to orthogonal and complementary methods, the ligand efficiency (LE) or one of its related metrics should ideally be used to keep track of the quality of follow-up ligands as they progress through the iterative optimization cycle. Some of these parameters are described below.

- Ligand Efficiency (LE) (Hopkins et al., 2004; Nissink, 2009; Davis and Roughley, 2017) $=\Delta$ G/HAC ${ }^{\text {A }}$;

- Binding Efficiency Index (BEI) (Abad-Zapatero, 2013) = $\mathrm{pKi} / \mathrm{MW}^{\mathrm{B}}$;

- Percentage Efficiency Index (PEI) (Abad-Zapatero, 2017; Davis and Roughley, 2017) =\% inhibition/MW B;

- Surface-binding Efficiency Index (SEI) (Abad-Zapatero, 2013) $=\mathrm{pIC}_{50} /$ (TPSA $^{\mathrm{C}}$ );

- Lipophilic Efficiency (LipE/LLE) (Shultz, 2013) $=$ pIC $_{50-}$ cLogP;

- Size-Independent Ligand Efficiency (SILE) (Nissink, 2009) = $\triangle \mathrm{G} / \mathrm{HAC}^{1-x}$;

- Ligand Efficiency-Dependent Lipophlicity (LELP) (Davis and Roughley, 2017) $=\log$ P/LE.

${ }^{\mathrm{A}}$ Heavy Atom Count; ${ }^{\mathrm{B}}$ Molecular Weight; ${ }^{\mathrm{C}}$ Topological Polar Surface Area;
For the sake of brevity these metrics will not be further discussed and we recommend the references above for a deeper understanding. The structural complexity of the protein makes larger, more complex and less efficient molecules less likely to bind. This is one of the main reasons why fragment libraries often yield more hits when compared to a drug-like molecule commonly used in HTS (Hann et al., 2001). The use of fragments is a bottom-up approach, starting from less complex molecules with greater binding efficiency and ending up with a larger optimized molecule. As already highlighted, there are three main strategies that can be employed to optimize a ligand found bound in its target surface: linking, merging and growing (Figure 3). The next sections are dedicated to discussing in more depth each of them.

\section{Growing}

Fragment growing (Figure 3A) is the strategy most commonly employed during FBLD campaigns. As the name suggests, it consists of modifying the fragments to increase their size. Conceptually this approach is identical to the traditional compound modification methods employed in the optimization of hits from HTS campaigns. This modification occurs through the addition of groups.

A recent paper published by Strecker et al. (2019) is an example of how the growing strategy can be used to improve bind affinity. Using computer-aided drug design (CADD) and synthesis, the authors explored small structural modifications in a previously (PDB: 3U0X) identified compound (1) $\left(\mathrm{K}_{\mathrm{i}}=800 \mu \mathrm{M}\right)$.

These studies showed that a modification of a fragment phenyl moiety to a naphthyl allowed two new simultaneous $\pi-\pi$ interactions, a parallel-displaced with $\operatorname{Trp} 300$ and an edge-to-face with His233. This minor modification led to a compound (2) with a 3-fold improved binding affinity $\left(K_{i}=271 \mu \mathrm{M}\right)$ (Figure 4). 
A

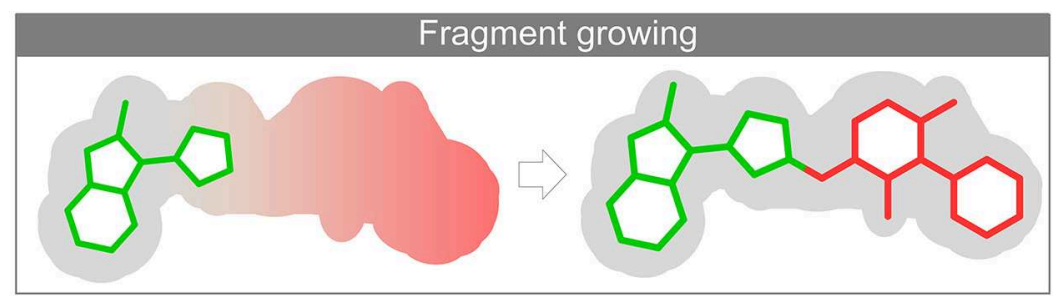

B

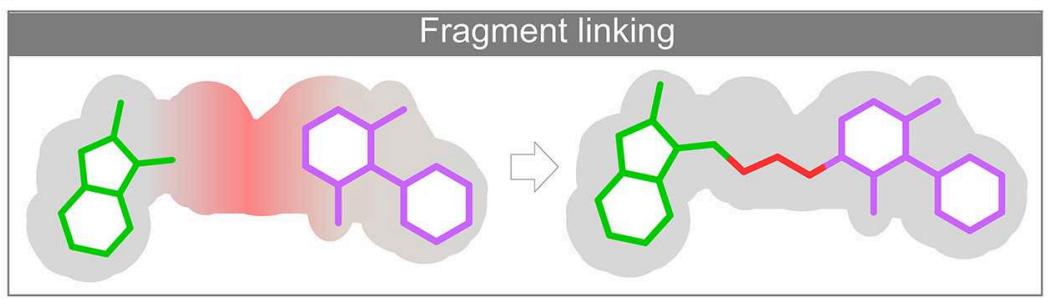

C
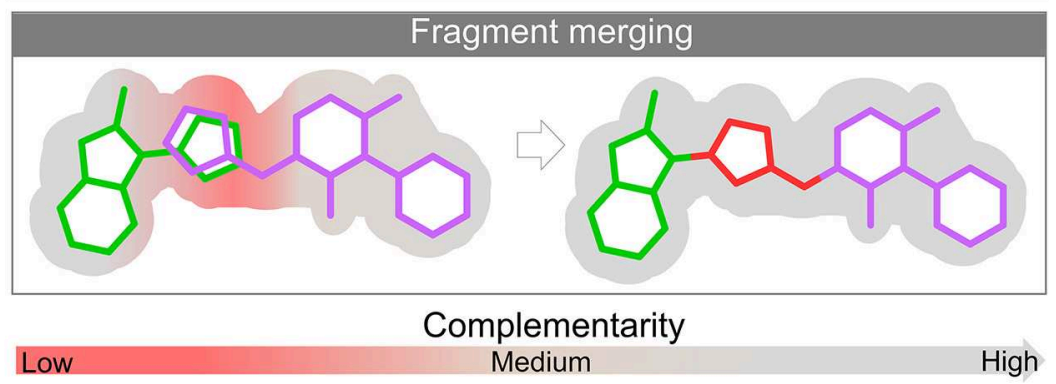

FIGURE 3 | Fragment optimization approaches: fragment growing (A), fragment linking (B), and fragment merging (C). The surface of the binding site is depicted in gray. The red and gray colors represent the level of complementarity of ligand with the active site. Pockets with low complementarity with ligand are colored in red; pockets with high complementarity with ligand are highlighted in gray.

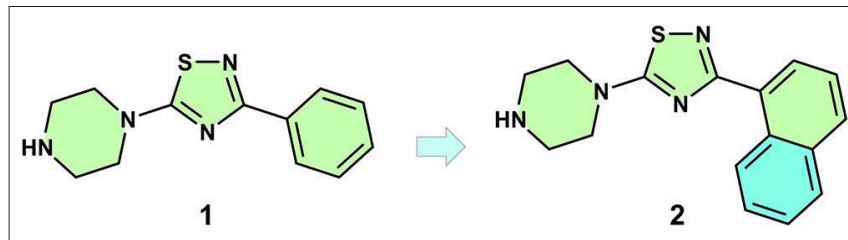

FIGURE 4 | Hit to lead progression of an initial fragment (1) to a compound (2) with improved affinity.

This example highlights the use of optimal growth vectors to introduce a rigid group, which led to an increased binding affinity. Alternatively, introduction of a moiety with increased number of rotatable bonds could impact negativelydue to the entropic penalty-in the affinity. Although this optimization approach can be computationally aided without further structural data, small modifications - as in the case of the hypothetical flexible moiety addition-can led to great changes in binding mode. When growing fragments is the chosen approach, structural data can be decisive to avoid misinterpretation.

\section{Linking}

Fragment linking (Figure 3B) describes the process of joining two non-competitive fragments (i.e., fragments that bind in two different sub-pockets of the binding site) with a chemical linker or spacer. Although conceptually simple, linking fragments is perhaps the most challenging strategy to implement. Although fragment linking is the most attractive approach in terms of rapid improvement of potency, the design of a linker with suitable flexibility while not disturbing the original binding modes of the fragments, makes it one of the most challenging optimization approaches.

As previously discussed, the introduction of flexible moieties affects these compounds properties and an optimal orientation should always be pursued. In fact, varying the degree of rigidity of a linker for the purpose of conformational restriction of the linked product can be used as a strategy for linker optimization, as it can be seen in Chung and colleagues work (Chung et al., 2009). This work shows how a conversion of oxime linkers into monoamine and diamines interferes with the rigidity and its impact on binding.

Although often neglected, the impact on the ADMET properties should also be taken in consideration. In the case of the linker, that usually adds rotatable bonds to the system (Ichihara et al., 2011; De Fusco et al., 2017), this modification can lead to poor PK features, like low permeability (Veber et al., 2002).

\section{Merging}

This strategy (Figure 3C) can be used in cases where two distinct fragments partially occupy the same region, or when two binding sites have regions in common and therefore their ligands are 
partially competitive with respect to the site. In such cases the overlapping parts form a nucleus where dissimilar parts come together. In a recent example, a gain of 2 orders of magnitude in potency was achieved for an inhibitor of flavindependent monooxygenase (EthA) transcriptional repressor (EthR) (Nikiforov et al., 2016) where the existence of overlapping groups within fragments bound to EthR allowed the use of merging as an optimization strategy.

Although not always possible, merging is a simpler strategy than linking, as there is no need to design a spacer that joins fragments together (Xu et al., 2017; Miyake et al., 2019). As also seen in this example, like linking (Davis and Roughley, 2017), this approach has the drawback of relying on high-quality structural data to go further in the optimization process.

Therefore, merging is an approach related to the "molecular hybridization" strategy, a long-consolidated approach in medicinal chemistry for designing new compounds with improved potency through the fusion of other active compound structures.

\section{IN SILICO STRATEGIES FOR F2L OPTIMIZATION}

\section{Hot Spots Analysis and Pocket Druggability Prediction}

Hot spots analysis is an important tool for structure-based F2L that allows the prediction of the small regions of the binding sites containing residues mostly contributing to the binding free energy (Cukuroglu et al., 2014). Once a fragment hit is experimentally identified, the hot spots analysis can be used to map the subsites around the fragment hit using small organic probes, driving the optimization into higher-affinity ligands (Hall et al., 2012).

One of the most used methods of hot spot analysis is the FTMap web server (Kozakov et al., 2015). This algorithm places 16 small organic probe molecules of different shape, size, and polarity on the protein surface to find favorable positions for each probe. Then, each probe type is clustered and overlapping clusters of different probes, called consensus sites (CSs), represent the hot spots. The consensus sites are ranked by the number of probe clusters, and the main hot spot is, generally, where the fragment hit binds and secondary hot spots are used to extend the fragment in the best direction (Hall et al., 2012; Ngan et al., 2012; Kozakov et al., 2015).

As an example, we used the FTMap server for predicting the hot spots for the oncogenic B-RAF kinase, the target of the first marketed drug from fragment-based drug design, vemurafenib (Bollag et al., 2012). Figure 5A shows the fragment hit experimentally bound to B-RAF kinase (PDB ID: 2UVX) (Donald et al., 2007), and the predicted hot spots around this fragment (shown in yellow dots) using the FTMap server. In Figures 5B-D, the iterative process of growing the fragment hit led to the discovery of the drug vemurafenib (PDB ID: 3OG7) (Bollag et al., 2010) with the hot spots shown in yellow dots. Although hot spot analysis was not used in the F2L process of vemurafenib, the results here showed that the predicted hot spots overlap the grown portions of vemurafenib.

During fragment screening, the fragment hits can bind in different sites of the protein (Giordanetto et al., 2019). If the binding site is not well-defined, the researchers can use the pocket druggability prediction to move forward in $\mathrm{F} 2 \mathrm{~L}$ with the most druggable site, capable to accommodate ligands orally bioavailable (Schmidtke and Barril, 2010; Hussein et al., 2015). There are many available methods for predicting pocket druggability and these are well-described and reviewed elsewhere (Barril, 2013; Abi Hussein et al., 2017).

\section{SAR by Catalog}

One fast and cheap way in F2L optimization is the SAR by catalog approach (Hall et al., 2017). This approach relies on the search of analogs of in-house or commercial databases that can be purchased or rapidly accessed for testing (Schulz et al., 2011). This process can use the fragment hit features for similarity, ligand-based pharmacophores, shape-based, fingerprints (Rogers and Hahn, 2010; Riniker and Landrum, 2013; Alvarsson et al., 2014), and substructure searches to find suitable compounds (Hubbard and Murray, 2011; Andrade et al., 2018). Some databases often used for SAR by catalog are ZINC (Sterling and Irwin, 2015), MolPort (https://www.molport.com), Mcule (https://mcule.com/), and eMolecules (https://www.emolecules. com) that contains collections of commercially available compounds. The databases Enamine (https://enaminestore. com), ChemDiv (http://www.chemdiv.com/) and ChemBridge (https://www.chembridge.com) are direct suppliers.

SAR by catalog approach only retrieves similar compounds or superstructures of the fragment hit. Thus, other filters should be applied to filter compounds with more optimized properties. These filters are molecular docking, ADMET, machine learning models, aqueous solubility, among others, and will be discussed later in this review.

\section{Molecular Docking}

Molecular docking is a computational approach used to predict the position, orientation, and the binding scores of small molecules to proteins (Torres et al., 2019). Hence, as the F2L process is commonly addressed as a combinatorial problem, molecular docking is a method that can be used in combination with other approaches to enhance the F2L process, and to increase the chances to convert a fragment hit into higher affinity ligands. The SAR by catalog approach in combination with molecular docking, for example, can be used to select compounds that maintain the fragment hit binding mode while the binding energy is optimized. Moreover, the number of generated optimized fragments can exceed the number that can be tested experimentally. Thus, applying molecular docking, large compound datasets are efficiently assessed using SAR by catalog, and a small subset of most promising compounds can be selected by binding modes and scores for experimental testing (Grove et al., 2016).

To overcome the problem that SAR by catalog has the limitation to cover only the finite chemical space of commercially available compounds (Hoffer et al., 2018), it is possible to 
A

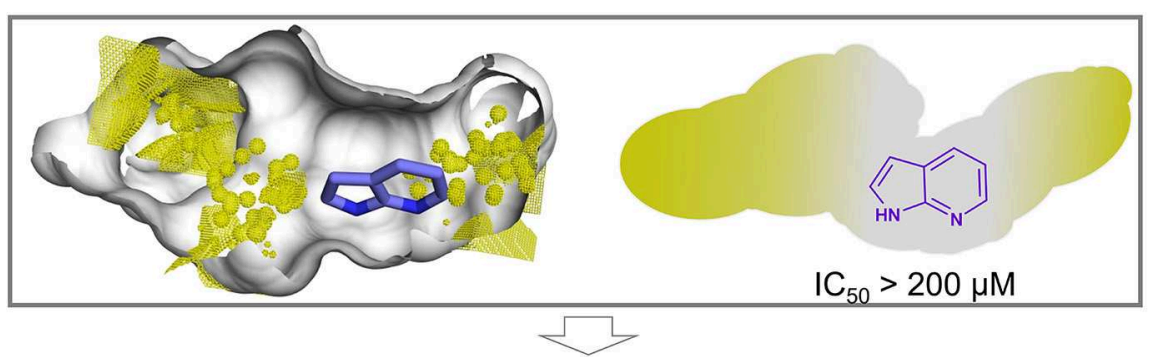

B

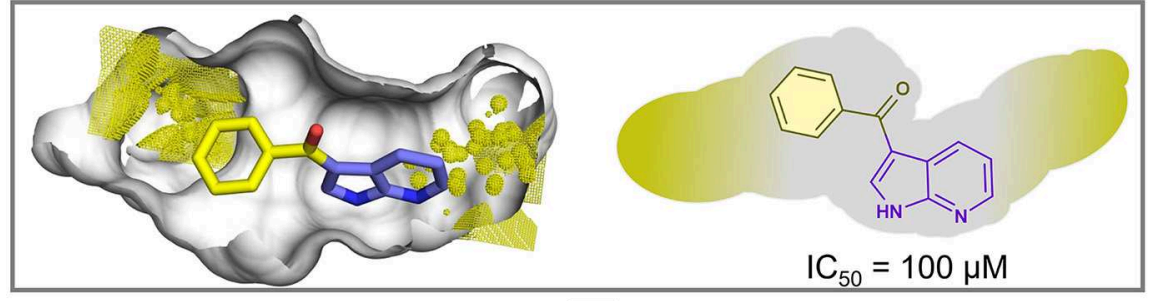

$\sqrt{2}$

C

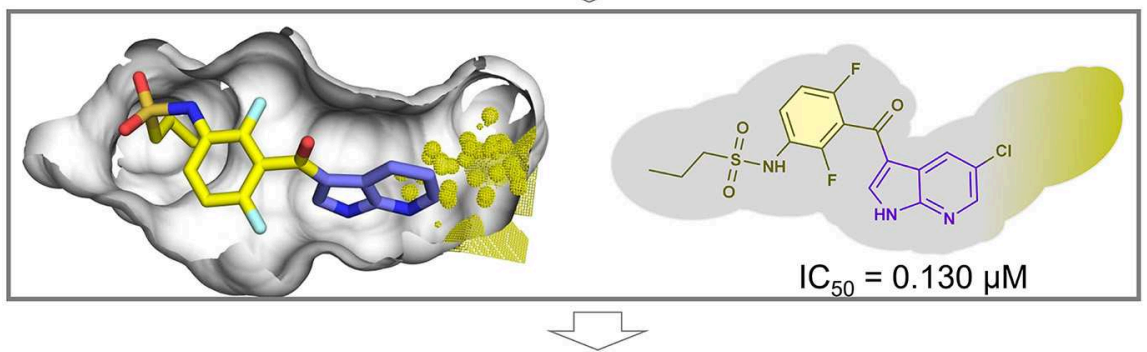

D

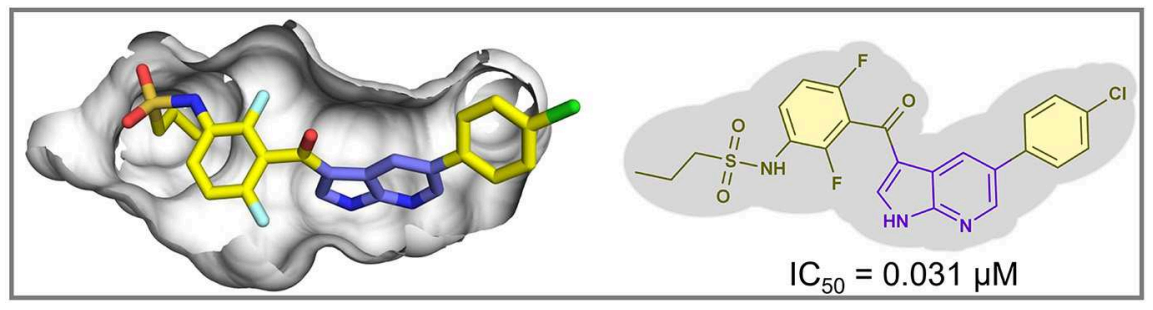

FIGURE 5 | Example of a hot spot analysis using FTMap web server of the oncogenic B-RAF kinase, the target of the first marketed drug from fragment-based drug design, vemurafenib. The surface of the binding site is depicted in gray. (A) (PDB ID: 2UVX) the fragment hit (carbon atoms in purple sticks) and the predicted hot spots (yellow dots and surface). (B-D) The iterative growing process of vemurafenib (PDB ID: 3OG7) overlapping the predicted hot spots (the carbon atoms of the fragment hit portion is shown in purple sticks and carbon atoms of the grown portions in yellow sticks).

generate virtual catalogs with analogs to hit fragments that can be easily synthesized, astronomically increasing the number of possible compounds. Then, a docking-based virtual screening can be applied to prioritize compounds for experimental evaluation (Rodríguez et al., 2016; Männel et al., 2017).

Another scenario in F2L is when the co-crystallization of a fragment hit commonly fails and no structural information about the binding mode is available. In these cases, alternative strategies for F2L process are required where the binding mode of a fragment can be predicted using molecular docking calculations (Kumar et al., 2012; Chevillard et al., 2018; Erlanson et al., 2019) on high-quality three-dimensional structures of the target in apo form or bound to other ligands. When neither of the latter are available, a theoretical model of the target protein can be obtained by homology modeling methods.
However, there are concerns about fragment docking in the scientific community. The assumption is that fragments, as low molecular weight compounds, are weak binders and promiscuous in binding modes, and consequently, the fragment docking implies in incorrect predictions of the binding modes. Also, there is a concern that scoring functions of the docking programs are parameterized to drug-like ligands, being inaccurate to differentiate native and other low-energy poses (Chen and Shoichet, 2009; Wang et al., 2015; Grove et al., 2016). To overcome these concerns, there are studies demonstrating no significant difference in docking performance between fragments and drug-like ligands (Verdonk et al., 2011; Joseph-mccarthy et al., 2013). They showed that molecular weight is not the principal parameter for docking performance, instead, for high LE compounds the docking performance fared better for both 
fragments and drug-like ligands (Verdonk et al., 2011; Kumar et al., 2012).

When available, the use of experimental structural information data can be used to support and improve docking performance. These data are used in docking programs including distance constraints, pharmacophore constraints, shape-based constraints, similarity or substructure overlap, interaction fingerprints, hydrogen-bond constraints, and others (Verdonk et al., 2011; Erlanson et al., 2019; Jacquemard et al., 2019).

Similarly to hot spot analysis, molecular docking can also be used to discover secondary binding pockets and guide the F2L process (Männel et al., 2017).

\section{Machine Learning (ML) and Deep Learning (DL) Models}

A large variety of F2L approaches use structure-based methods to optimize fragments into high-affinity ligands taking into consideration the steric and electronic constraints within binding pockets of the target of interest (Schneider and Fechner, 2005). However, the optimized compounds generated constantly present drawbacks of poor synthetic feasibility and/or undesirable biological properties, including absorption, distribution, metabolism, excretion, and toxicity (ADMET) properties (Yang et al., 2019b). In the last years, novel ligandbased methods, including machine learning (ML) models, have been used for F2L campaigns. ML models are statistical methods that present the capacity to learn from data without the explicit programming for this task, and then, make a prediction for new compounds (Mak and Pichika, 2019). The increase of storage capacity and the size of the datasets available, coupled to advances in computer hardware such as graphical processing units (GPUs) (Gawehn et al., 2018), provided means to move theoretical studies in ML to practical applications in drug discovery (Vamathevan et al., 2019).

The ML algorithms are widely used to construct quantitative structure-activity relationship (QSAR) models, able to find mathematical correlations between molecular features and compound activity/property, and this correlation can be categorical (active, inactive, toxic, nontoxic, etc.) or continuous ( $\mathrm{pIC}_{50}, \mathrm{pEC}_{50}, \mathrm{Ki}$, and others) by means of classification or regression techniques (Tropsha, 2010; Cherkasov et al., 2014). Thus, machine learning-based QSAR models can be constructed for biological activity, ADMET properties, solubility and synthetic feasibility, among other endpoints, and applied after fragment optimization, with aforementioned methods, in a cascade virtual screening for filtering compounds with the desired activities and properties (Figure 6) (Braga et al., 2014; Neves et al., 2018; Pérez-Sianes et al., 2018).

More recently, a subfield of ML called deep learning (DL) which utilizes artificial neural networks to learn from a large amount of data have been used to resolve complex problems (Mak and Pichika, 2019). DL models are not only able to learn from a dataset and to make predictions for new data but are also able to generate new data instances through a constructive process (Schneider, 2018). In this context, there has been a rising interest in using DL generative and predictive models for F2L optimization (Olivecrona et al., 2017; Gupta et al., 2018).

For this task, a combination of DL architectures is used and in many cases, generative DL models based on recurrent neural networks (RNNs) are trained on the simplified molecular input line entry system (SMILES) representation of compounds from large databases (DrugBank, ChEMBL, etc.) to learn the syntax of SMILES language and the chemical space distribution (Olivecrona et al., 2017). After training, the models are able to generate new strings that are new SMILES, corresponding to new compounds (Segler et al., 2018). Then, the transfer learning (TL) can be used to fine-tuning the model and generate compounds related to a fragment hit. As the name suggests, TL learns and transfers the information from an old source to a new application (Yang et al., 2019b). The aim of this integrative approach is to learn general features from a big dataset and, then, retrain the model focusing on a smaller dataset such as fragment hits, for F2L purposes (Figure 7) (Gupta et al., 2018; Segler et al., 2018). Gómez-Bombarelli et al. used variational autoencoder (VAE) to encode SMILES into a continuous latentspace, then a separate multilayer perceptron trained to predict several properties on the latent space was applied to generate new molecules with the desired properties. After this, a decoder was used to retrieve the molecules on the latent space into SMILES (Gómez-Bombarelli et al., 2018). Handling these DL methods in a multidimensional way, fragment hits can be optimized automatically taking into consideration several parameters such as bioactivity, solubility, synthetic feasibility, and ADMET properties, generating new compounds with optimized values for these parameters (Figure 7) (Olivecrona et al., 2017; Ramsundar et al., 2017; Gómez-Bombarelli et al., 2018; Harel and Radinsky, 2018; Li et al., 2018; Merk et al., 2018; Polykovskiy et al., 2018; Popova et al., 2018; Putin et al., 2018; Awale et al., 2019; Vamathevan et al., 2019).

\section{De novo Design}

The de novo approach looks for new chemical entities from scratch within a structurally defined binding site (Schneider and Clark, 2019). These entities are generated out of building blocks, either by growing from an initial fragment or by linking two or more non-overlapping fragments (Dey and Caflisch, 2008; Kumar et al., 2012). Since their arise, in silico methods have played an important role in FBDD (Kumar et al., 2012).

\section{Software for Building New Compounds Within a Structurally-Defined Binding Site}

De novo design software takes advantage of a known binding mode of a fragment, described experimentally or computationally, to propose modified analogs with improved binding affinities. The LUDI (Bohm, 1992) program was one of the first programs developed for de novo design. It calculates the interaction sites, maps the molecular fragments, and connects them using bridges, using an empirical scoring. Considering the vast chemical space, evolutionary algorithms are widely used (Srinivas Reddy et al., 2013). In this context, the program GANDI (Dey and Caflisch, 2008) connects pre-docked fragments with linker fragments using a genetic algorithm and a tabu search. 


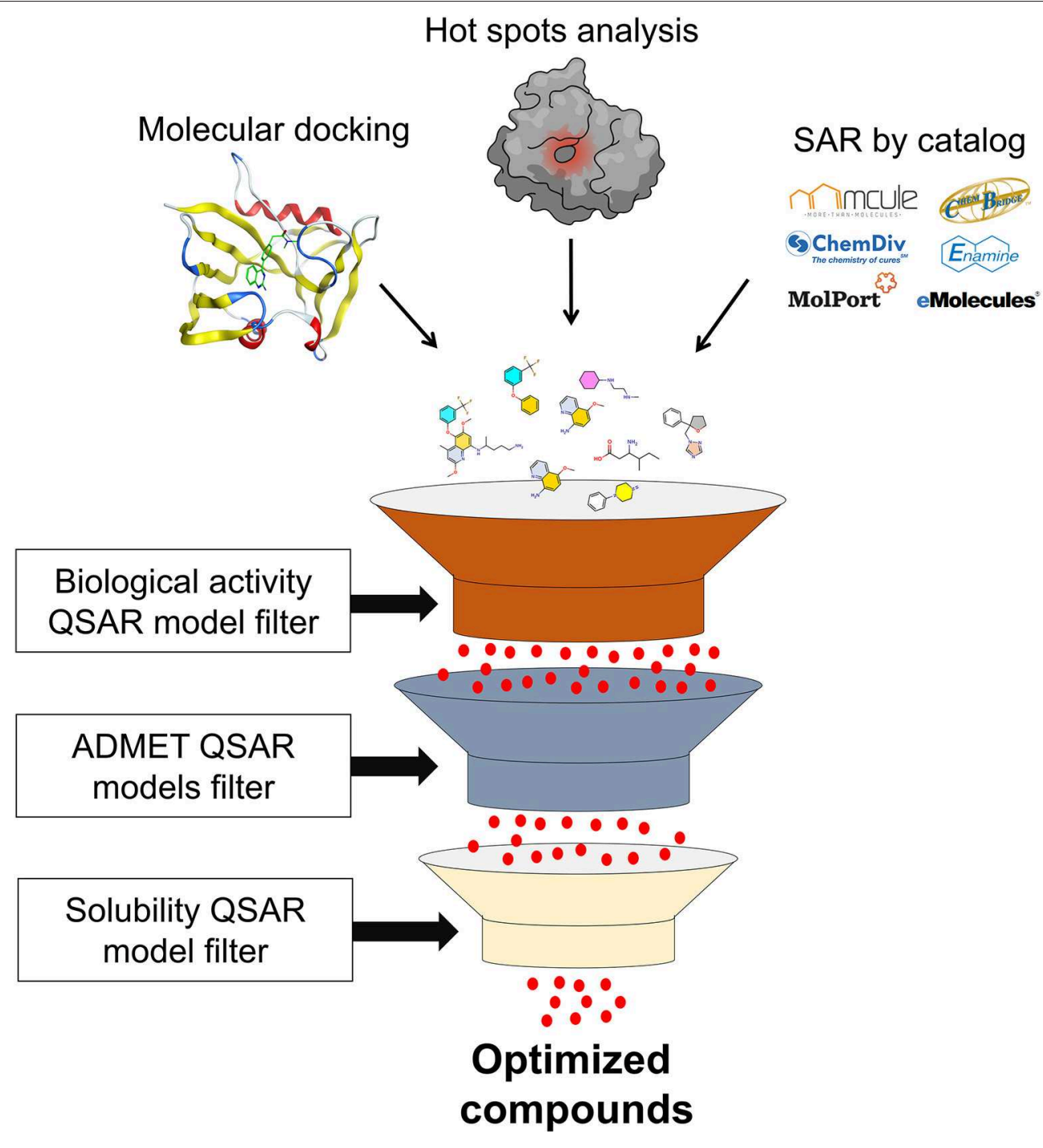

FIGURE 6 | Cascade virtual screening filtering optimized compounds with the desired activities and properties.

The scoring function is a linear combination of force-field binding energy and similarity measures. BREED (Pierce et al., 2004) is a computational method for merging fragments that is widely used. It aligns the $3 \mathrm{D}$ coordinates of two ligands and recombines the fragments or substructures into the overlapping bonds to generate new hybrid molecules in a strategy called fragment shuffling. LigBuilder (Wang et al., 2000; Yuan et al., 2011 ) is a program that uses a genetic algorithm to build up the ligands using a library of organic fragments. It contemplates the growing and linking approach. The 2.0 version includes the synthesis accessibility analysis through a chemical reaction database and retro-synthetic analysis. Autogrow (Durrant et al., $2009,2013)$ is another growing approach algorithm that builds a fragment upon a "core" scaffold. The fragment is docked to the receptor. A genetic algorithm evaluates the docking score to select the best population which forms the subsequent generation. The last version considers the synthetic accessibility and the druggability. The program ADAPT (Pegg et al., 2001; Srinivas Reddy et al., 2013) applies a genetic algorithm which uses molecular interactions and docking calculations as a fitness function to reduce the search space. The initial sets of compounds are iteratively built until it reaches the predefined target value.

\section{Prediction of ADMET Properties of New Compounds}

The ADMET properties and synthetic accessibility (SA) constitutes the secondary constraints whereas primary constraints are geometric and chemical constraints derived from the receptor or target ligand(s) and internal constraints to the geometry and chemistry of the lead compound being constructed. Issues with these points result in the majority of clinical trial failures (Dong et al., 2018). Numerous software and web platforms were developed to predicted ADMET parameters but presented limitations due to narrow chemical 


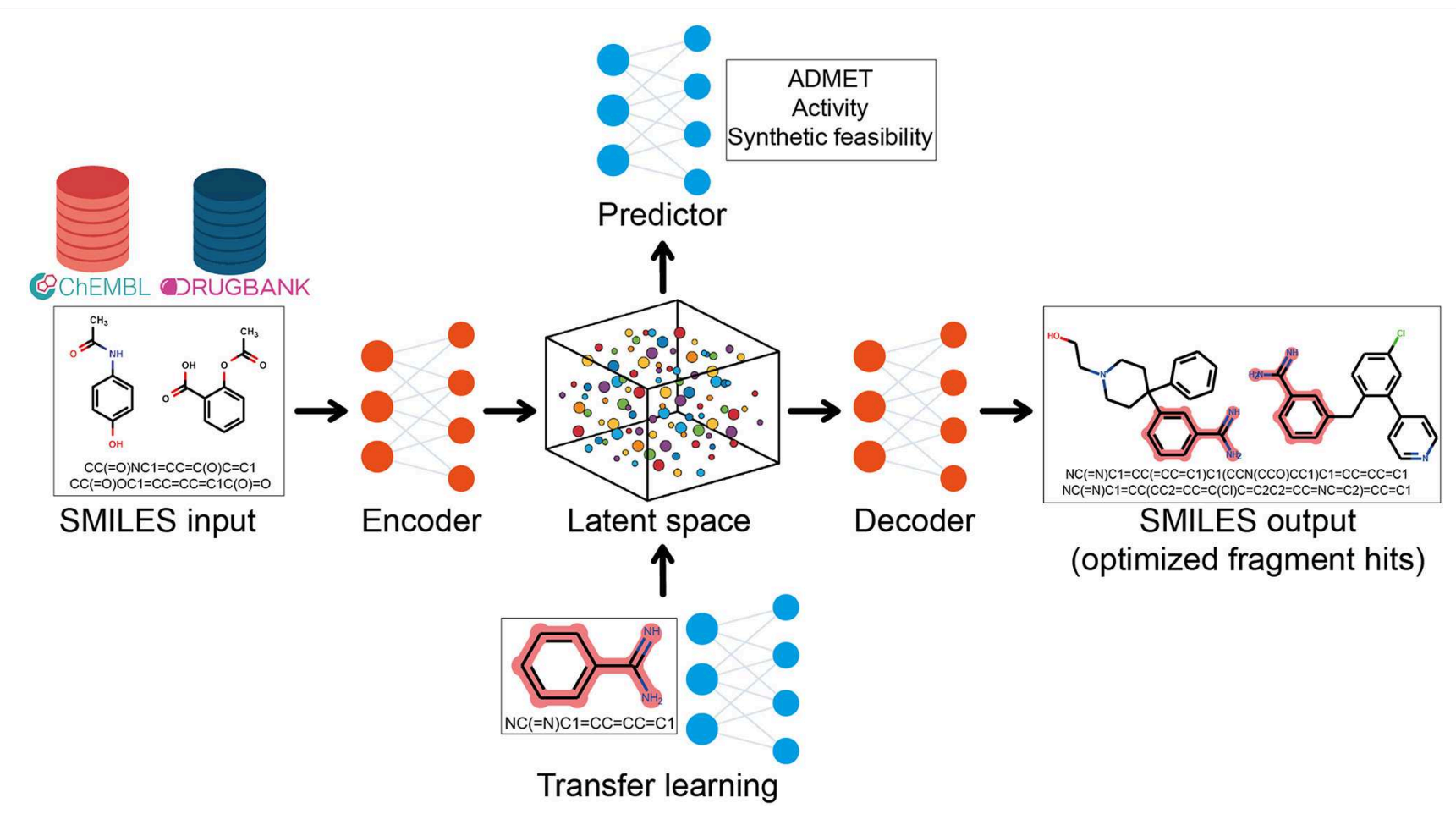

FIGURE 7 | Representation of the integrative approach of generative and predictive deep learning models and transfer learning for fragment-to-lead optimization.

space coverage or expensive prices (Cheng et al., 2012). Recent works predominantly rely on ML methods, like random forest (RF), support vector machine (SVM), and tree-based methods (Ferreira and Andricopulo, 2019). The vNN Web Server for ADMET predictions (Schyman et al., 2017) is a publicly available online platform to predict ADMET properties and to build new models based on the k-nearest neighbor (k-NN), which rest on the premise that compounds with similar structures have similar activities. vNN uses all nearest neighbors that are structurally similar to define the model's applicability domain. The similarity distance employed is Tanimoto's coefficient. The platform allows running pre-build ADMET models, and to build and run customized models. Those models assess cytotoxicity, mutagenicity, cardiotoxicity, drug-drug interactions, microsomal stability, and likelihood of causing drug-induced liver injury. Like all machine learning methods, the lack of training data is a limitation.

Pred-hERG (Braga et al., 2015; Alves et al., 2018) is a web app that allows users to predict blockers and non-blockers of the hERG channels, and important drug anti-target associated with lethal cardiac arrhythmia (Mitcheson et al., 2000). The current version of the app (v. 4.2) was developed using ChEMBL (Willighagen et al., 2013) version 23, containing 8,134 compounds with hERG blockage data after curation, using robust and predictive machine learning models based on RF. This app is publicly available at http://labmol.com.br/predherg/.

In admetSAR 2.0 (Cheng et al., 2012; Yang et al., 2019a) tool, the predictive models are built using RF, SVM and kNN algorithms. It presents 27 endpoints and also includes ecotoxicity models and an optimization module called ADMETopt that optimize the query molecule by scaffold hopping based on ADMET properties. The ADMETlab platform (Dong et al., 2018) performs its evaluations based on a database of collected entries and assess drug-likeness evaluation, ADMET prediction, systematic evaluation and database/similarity searching. It uses 31 endpoints applying RF, SVM, recursive partitioning regression $(\mathrm{RP})$, naive Bayes (NB), and decision tree (DT).

SwissADME tool (Daina et al., 2017) uses predictive models for physicochemical properties, lipophilicity and water solubility. It also analyses pharmacokinetics models as BBB permeability, gastrointestinal absorption, $\mathrm{P}$-gp binding, skin permeation $(\operatorname{logKp})$, and CYP450 inhibition. Additionally, the tool presents five drug-likeness models (Lipinsky, Ghose, Veber, Egan, and Muegge) and medicinal chemistry alerts. It is integrated with the SwissDrugDesign workspace. The QikProp (Schrödinger, LLC, NY, 2019) provides rapid predictions of ADME properties for molecules with novel scaffolds as for analogs of well-known drugs and display information about octanol/water and water/gas $\log$ Ps, $\log$ S, $\log \mathrm{BBB}$, overall CNS activity, Caco-2 and MDCK cell permeabilities, log Kd for human serum albumin binding, and $\log \mathrm{IC}_{50}$ for HERG $\mathrm{K}+$-channel blockage.

\section{Prediction of Synthetic Tractability (Synthesizability) of New Compounds}

Even though large numbers of molecules are generated by de novo design, many of them are synthetically infeasible 
(Dey and Caflisch, 2008). To address this problem, methods to calculate the synthetic accessibility (SA) are being developed. SA can be addressed by estimating the complexity of the molecule or making a retrosynthetic approach, where the complete synthetic tree leading to the molecules needs to be processed (Ertl and Schuffenhauer, 2009). SYLVIA (Boda et al., 2007) is one of the programs that estimate the synthetic accessibility of an organic compound. It obtains the SA score by the addition of five variables as the molecular graph complexity, ring complexity, stereochemical complexity, starting material similarity and reaction center substructure, where the first three are structure-based and the other two utilize information from starting material catalogs and reaction databases. Ertl and Schuffenhauer (2009) developed another method that uses historical synthetic knowledge obtained by analyzing information from millions of already synthesized chemicals and also considers molecule complexity. The method is based on a combination of fragment contributions and a complexity penalty. Podolyan et al. (2010) presented two approaches to quickly predict the synthetic accessibility of chemical compounds by utilizing SVMs operating on molecular descriptors. The first approach (RSsvm) identifies compounds that can be synthesized using a specific set of reactions and starting materials and builds the model by training the compounds identified as synthetically or otherwise accessible by retrosynthetic analysis while the second approach (DRSVM) is constructed to generate a more general assessment. More recently, Fukunishi et al. (2014) designed a new method of predicting SA based on commercially available compound databases and molecular descriptors where the SA is estimated from the probability of the existence of substructures of the compound, the number of symmetry atoms, the graph complexity, and the number of the chiral center of the compound.

\section{Synthesizability-Aware Methods}

Given the difficulty of synthesis of most of the leads produced by de novo approaches, some programs added methods to score the SA. Lead+Op (Lin et al., 2018) is an example of these programs that takes an initial fragment, looks for associated reaction rules, virtually generate the reaction products and select the best binding conformation. Them it generates conformers and select one that becomes a reactant for another round. Also, programs mentioned above as LigBuilder and Autogrow include SA analysis on their current versions. In the medicinal chemistry component of SwissADME, a SA score is also included.

Different programs use distinct algorithms for de novo design compounds in CADD. Table 1 summarizes some programs cited in this section.

\section{CASE STUDIES IN THE LAST FIVE YEARS Case 1: FBDD in the Development of New Anti-mycobacterium Drugs}

A successful application of the FBDD techniques have been applied to early stage drug discovery of new therapeutics against Mycobacterium sp. and in particular $M$. tuberculosis (Mtb) and M. abscessus (Mab) (Thomas et al., 2017). Mtb,
TABLE 1 | FBDD programs with respective approaches.

\begin{tabular}{|c|c|c|c|}
\hline Program & Algorithm & $\begin{array}{l}\text { FBDD } \\
\text { Approach }\end{array}$ & SA \\
\hline AUTOGROW & $\begin{array}{l}\text { Docking + Genetic } \\
\text { Algorithm }\end{array}$ & Growing & $\begin{array}{l}\text { YES (Latest } \\
\text { version) }\end{array}$ \\
\hline LUDI & Empirical scoring & Linking & NO \\
\hline AUTO $T$ and $T$ & $\begin{array}{l}\text { Transplants fragments into } \\
\text { the lead }\end{array}$ & Merging & NO \\
\hline LeadOp+R & $\begin{array}{l}\text { Looks for associated } \\
\text { reaction rules }\end{array}$ & Growing & YES \\
\hline GANDI & Genetic Algorithm & Linking & NO \\
\hline LigBuilder 2 & Genetic Algorithm & $\begin{array}{l}\text { Linking and } \\
\text { Growing }\end{array}$ & YES \\
\hline ADAPT & Genetic Algorithm & Growing & NO \\
\hline
\end{tabular}

the causative agent of tuberculosis, has several therapeutic interventions developed to treat the disease. However, through their long-term use and misuse, the efficacy of these drugs is becoming reduced with strains currently circulating that are mono-resistant, multidrug-resistant, extensively drug-resistant and totally drug-resistant. Despite this little drug development activity has been undertaken since the 1960's. However, relatively in response to the growing drug-resistant threat many different approaches are being deployed to developing novel therapeutics, including FBDD. An example of such an effort is against the meta cleavage product hydrolase (HsaD) that is involved in cholesterol catabolism in $M t b$. Initial screening was conducted on a library of 1,258 fragments using differential scanning fluorimetry, with hits confirmed by ligand-observed NMR spectroscopy and inhibition by enzymatic assay. The three confirmed fragment initial hits were structurally characterized by X-ray crystallography and fragment soaking. A small series of compounds based on these hits were further tested for activity both in vitro and ex vivo with promising results (Ryan et al., 2017).

Another target of $M t b$ where FBDD has been applied is the pantothenate synthetase (Pts) where a similar sized fragment library of 1,250 rule-of-three compliant fragments was investigated. An initial screen was performed using a thermal shift assay, followed by a secondary screen using 1-D NMR spectroscopy with ultimate hit validation by isothermal titration calorimetry and characterization by X-ray crystallography. Three distinct fragment binding sites were identified (Silvestre et al., 2013). Follow-up expansion of one of the fragment sites using a combination of fragment linking and fragment growing generated a new series of inhibitors. Though fragment linking seemed to be an attractive approach, the limitation in the repertoire of linkers compromised the binding mode. Greater success came from fragment growth using expert knowledge and the protein target as a template (Hung et al., 2009).

Targets in other pathogenic Mycobacterium sp. have also been subject to successful FBDD campaigns. Most notably the recent development of inhibitors against tRNA methyltransferase (TrmD) of $M$. abscessus (Mab). This multi-drug resistant pathogen is increasingly problematic in individuals with cystic 
fibrosis and other chronic lung conditions. A library of 960 fragments was screened biophysically using differential scanning fluorimetry in a similar fashion used for HsaD, with 53 hits taken to validation and structural characterization using X-ray crystallography (no NMR based validation was undertaken). Only 27 fragments could be validated all of which bound within the substrate binding pocket. A strategy of fragment-merging centered around the overlap of a 4-methoxyphenyl ring system with the indole ring system of two fragments that spanned the adenine and ribose binding pockets. This was explored successfully with a new combined compound providing a new aminopyrazole-indole scaffold with both improved affinity $\left(K_{\mathrm{d}}=\right.$ $110 \mu \mathrm{M}, \mathrm{LE}$ of 0.36 ) and prospects for further elaboration relative to the parent fragments. It also exhibited inhibition activity in vitro and ex vivo with promising in vivo activity also against $M$. leprae, the causative agent of leprosy (Whitehouse et al., 2019).

These successful FBDD campaigns against a range of targets in pathogenic Mycobacterium have yielded promising leads with indications of efficacy in ex vivo and in vivo demonstrating both the power and efficacy of the approach. The ability of these leads to work across a range of pathogens is also highly encouraging. However, work still needs to be done to improve these leads to progress them into early clinical evaluations and into clinical use.

\section{Case 2: Inhibitors of Dengue Virus Enzymes}

A 2014 paper (Coutard et al., 2014) describes the use of FBLD in the discovery of inhibitors for an important subunit of dengue virus (DENV) viral replication complex. In this work, 500 fragments were screened against two subunits of the viral replication complex: NS3 helicase (Hel) and the NS5 mRNA methyltransferase (MTase) subunits. DENV Hel, located in the $C$-terminal region of the NS3 subunit of the replication complex, is involved in viral genome replication and RNA capping. The role of DENV NS5 MTase is related with a double methylation (N-7 and 2'-O) during the cap formation process in flavivirus (Dong et al., 2008).

The authors used a combination of Thermal Shift Assay (TSA), X-ray diffraction crystallography (XRD) and enzymatic assays in order to screen compounds against NS3 DENV Hel and NS5 DENV MTase subunits. The TSA was used as the primary screening technique. During the TSA screening, not surprisingly part of the fragments-used at high concentrations and with poorly optimized physicochemical properties-presented solubility problems. This was the reason for the exclusion of $\sim 4.8 \%$ of the screened compounds during this phase. This initial screening yielded 68 hits, from those, 7 were found bound to the DENV MTase subunit by XRD.

Using a direct colorimetric ATPase-based assay to identify inhibitors, from those previous 7 crystallographic hits, 5 fragments (Table 2) were classified as hits with their potency varying between $180 \mu \mathrm{M}$ and $9 \mathrm{mM}$.

In the most recent work, the fragments $\mathbf{3}$ and $\mathbf{4}$ (Figure 8) were found bound at the DENV MTase S-Adenosyl-L-methionine (AdoMet) binding site using XRD. A computer-aided fragment optimization gave rise to a new series of compounds using these
TABLE 2 | Inhibition and potency data from the final hits (Coutard et al., 2014).

\begin{tabular}{lcc}
\hline $\begin{array}{c}\text { DENV 2'O-Mtase activity } \\
\text { inhibition (\%) }\end{array}$ & $\begin{array}{c}\text { DENV 2'O-MTase } \\
\text { activity IC } \text { (50 }^{(\mathbf{m M})}\end{array}$ \\
\hline 81 & 4 & $3.90 \pm 0.16$ \\
91 & 11 & $2.83 \pm 0.18$ \\
95 & 85 & $0.18 \pm 0.01$ \\
157 & 9 & $9.39 \pm 0.90$ \\
217 & 11 & $3.12 \pm 0.27$ \\
\hline
\end{tabular}

two fragments. The urea was used as a linker to connect the fragments. Further modifications yielded compounds $\mathbf{5}$ and $\mathbf{6}$ (Figure 8).

During the optimization process, the authors had good insights about the important features to the molecule binding on this target. One of these features is the presence of phenyl rings substituted in meta position and is crucial for favoring binding.

This work yielded two inhibitors (5 and 6) with potency around $100 \mu \mathrm{M}$, even though no effect was observed on a cell assay. Despite this negative result, this work showed the feasibility of the FBDD approach in getting micromolar inhibitors from structurally simple fragments.

\section{Case 3: MTH1 Inhibitors for Anticancer Drug Discovery}

The mutT homolog 1 (MTH1) is an enzyme involved in the prevention of incorporation of deoxynucleoside triphosphates (dNTPs) oxidized by reactive oxygen species (ROS), e.g., 8-oxodGTP or 2-OH-dATP, into DNA, which prevents the killing of the cell. MTH1 is frequently overexpressed in cancer cells and is non-essential in normal cells, proving to be a druggable target for cancer treatment (Smits and Gillespie, 2014; Berglund et al., 2016).

Rudling et al. applied a combination of molecular docking, SAR by catalog, and experimental testing for discovering and optimizing MTH1 inhibitors (Rudling et al., 2017). Initially, a molecular docking-based virtual screening using a crystal structure of MTH1 was performed using 0.3 million fragments from the ZINC fragment-like database, all commercially available. Subsequently, for the 5,000 top-ranked fragments, allowed the search of analogs representing superstructures of the fragment or containing similar substructures in the ZINC database using the chemical structures encoded as circular fingerprints and the Tversky similarity index (Tversky, 1977). The criteria used to select analogs from 4.4 million commercially available compounds in the ZINC database was the following: (i) Tversky similarity $>0.8$; (ii) up to six additional heavy atoms (HAs) compared to the parent fragment; (iii) improved docking score $80 \%$ lower compared to the parent fragment; (iv) visual inspection of the binding modes. After these analyses, a set of 22 commercially available fragments with at least five analogs comprising the above-mentioned criteria were selected for experimental evaluation. Five of these 22 fragments showing $\mathrm{IC}_{50}$ values ranging from 5.6 to $79 \mu \mathrm{M}$ were considered hits and were used for F2L (Table 3). The fragment 7 presented an 


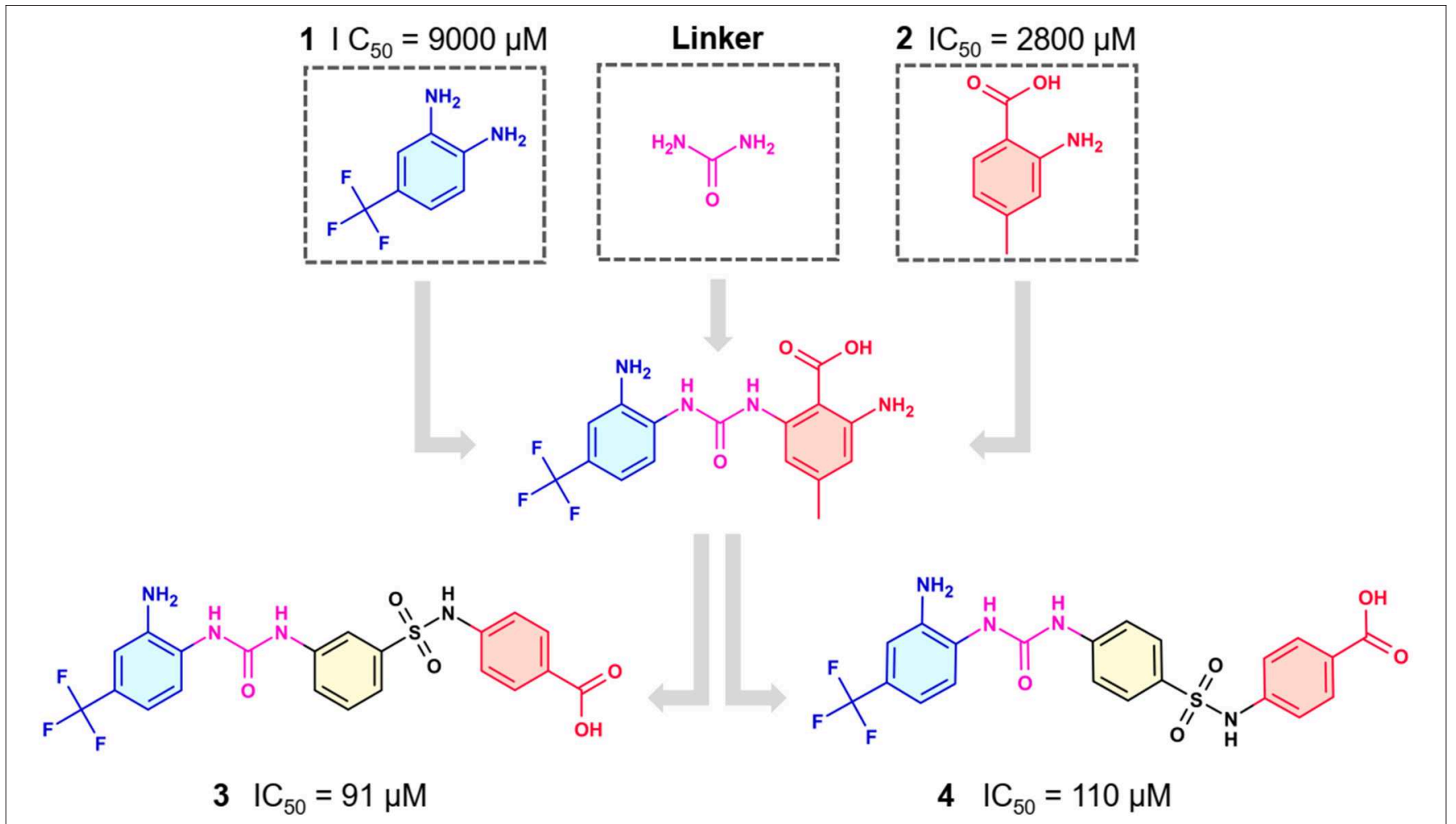

FIGURE 8 | Fragment optimization predecessors and products (Coutard et al., 2014; Benmansour et al., 2017).

$\mathrm{IC}_{50}$ value of $79 \mu \mathrm{M}$ and its most potent analog presented an $\mathrm{IC}_{50}$ of $170 \mathrm{nM}$, representing a 470 -fold improvement (Table 3). Although the crystal structure of the fragment 7 in complex with MTH1 was not obtained, the crystallization of its most potent analog and MTH1 was solved at $1.85 \AA$ resolution, demonstrating an RMSD of $0.6 \AA$ between the common atoms of the fragment 7 (binding mode predicted by molecular docking) and the analog (crystal structure). Because of the closely related structures and binding modes of fragments $\mathbf{8}$ and $\mathbf{9}$, its analogs were analyzed together, but the most potent analog presented an $\mathrm{IC}_{50}$ of only $3.5 \mu \mathrm{M}$. The most potent analog of fragment 10 presented a 190fold increase of the activity, with an $\mathrm{IC}_{50}$ of $120 \mathrm{nM}$ (Table 3). The crystallization of fragment $\mathbf{1 0}$ with MTH1 was also unsuccessful, but the molecular docking was able to predict the binding mode, showing an RMSD of 0.9 comparing the overlapping atoms with the crystal of the most potent analog obtained at $1.50 \AA$ resolution. The analogs of the fragment $\mathbf{1 1}$ were not available during the study. This work demonstrated that virtual screening and SAR by catalog can be used to rapidly identify and optimize fragments into nanomolar inhibitors (Rudling et al., 2017).

\section{Case 4: New Acetylcholinesterase Inhibitors Against Alzheimer's Disease}

Alzheimer's disease is a neurodegenerative disorder and has no cure. The actual treatments are based on drugs that leverage the transmission of electrical impulses. Pascoini et al. (2019), computationally developed new acetylcholinesterase (AChE) inhibitors. AChE is responsible for decreasing levels of acetylcholine in the synaptic cleft. Their inhibition enhances the transmission of the electric impulse (Polinsky, 1998; Talesa, 2001).

For the in silico inhibitor development, they divided the process into four steps. First, a de novo design was applied to generate an initial library of compounds. The first library was then filtered according to ADME properties at the second step. In the third step, the filtered library was filtered again using a similarity criterion. Finally, the resulting library was used in docking studies. The best three complexes were used for molecular dynamic studies. In this work, they used three reference drugs for $\mathrm{AD}$ treatment: donepezil, galantamine, and rivastigmine.

For the de novo design, the LigBuilder software was used. The CAVITY procedure was employed to detect and analyze ligand-binding sites of the target. It classified the cavities' druggability, who would be used for docking studies. The BUILD procedure was used in the exploring and growing/linking modes. In the explore mode, fragments from the program's database were added in the protein site and their interaction was scored. Then, the fragments with the best scores were linked. In the growing mode, seeds molecules were put at the binding site and fragments were added to the seeds. At the linking mode, the seed was divided into fragments and other fragments were added to them. After the BUILD procedure, they got a library of 2.5 million compounds. The resulting library was filtered according to ADME properties with the software QUIKPROP where molecules that infringed more than five properties (physicochemical properties, lipophilicity, water solubility, pharmacokinetics models) were discarded. A library 
TABLE 3 | Experimental data for the five most potent MTH1 inhibitors (data taken from Rudling et al., 2017).

\begin{tabular}{|c|c|c|c|c|}
\hline Fragment ID & Fragment 2D structure & Fragment $\mathrm{IC}_{50}$ & $\begin{array}{l}\text { Most potent analog 2D } \\
\text { structure }\end{array}$ & Analog $\mathrm{IC}_{50}$ \\
\hline 7 & & $79 \mu \mathrm{M}$ & & $0.17 \mu \mathrm{M}$ \\
\hline 8 & & $24 \mu \mathrm{M}$ & & $3.5 \mu \mathrm{M}$ \\
\hline 9 & $\mathrm{NH}_{2}$ & $26 \mu \mathrm{M}$ & $\mathrm{n} / \mathrm{a}$ & $\mathrm{n} / \mathrm{a}$ \\
\hline 10 & & $23 \mu \mathrm{M}$ & & $0.12 \mu \mathrm{M}$ \\
\hline
\end{tabular}

n/a, not available.

of 6,000 compounds results from this process. After this, the Tanimoto's coefficient was applied to measure the similarity among the molecules. Molecules below 0.85 were excluded and 1,500 molecules were considered for the next step. The final step consisted of docking studies, carried out with the GLIDE software and the Induced Fit Docking protocol. Afterward, they selected the three best complexes from the docking and used them as input structures for molecular dynamic studies. Finally, they obtained three compounds with high stability and good binding energies, some of them even better than the reference drugs.

\section{CONCLUDING REMARKS}

FBDD has matured to become a key strategy in modern pharmaceutical research. With less requirement for large chemical libraries and the possibility of using a range of biophysical methods for screening, the easier and scalable implementation of this strategy has facilitated its popularization, especially among academic institutions and smaller pharmaceutical companies.

The main reason for the success of the FBDD strategy is because it presents a more efficient and consistent route for optimization of initial screening hits into lead compounds. As reviewed here, many routes are available for expansion of fragment hits and in silico methods are key to support or guide the majority of them.

A variety of in silico methods have been used in F2L optimization in FBDD, from binding site analysis to de novo design of new fragment-derived ligands with synthesizabilityaware methods. The case studies highlighted here clearly 
demonstrate how the different in silico methods can be used in integrated form and combined with experimental approaches to successfully develop higher affinity ligands from fragments.

Advances in artificial intelligence methods, such as deep learning, hold a great potential to accelerate the optimization of fragment hits in lead compounds. Recent examples show that these hits can be already optimized automatically taking into consideration several parameters such as bioactivity, solubility, synthetic feasibility, and ADMET properties.

\section{AUTHOR CONTRIBUTIONS}

FS, CA, and NF drafted the review topics with the abstract and revised the manuscript. FS drafted the conclusion and

\section{REFERENCES}

Abad-Zapatero, C. (2013). Ligand Efficiency Indices for Drug Discovery. Oxford, UK: Elsevier.

Abad-Zapatero, C. (2017). Are SAR tables obsolete? Drug Discov. Today 22, 195-198. doi: 10.1016/j.drudis.2016.12.002

Abi Hussein, H., Geneix, C., Petitjean, M., Borrel, A., Flatters, D., and Camproux, A.-C. (2017). Global vision of druggability issues: applications and perspectives. Drug Discov. Today 22, 404-415. doi: 10.1016/j.drudis.2016. 11.021

Alvarsson, J., Eklund, M., Engkvist, O., Spjuth, O., Carlsson, L., Wikberg, J. E. S., et al. (2014). Ligand-based target prediction with signature fingerprints. J. Chem. Inf. Model. 54, 2647-2653. doi: 10.1021/ci500361u

Alves, V., Braga, R., Muratov, E., and Andrade, C. (2018). Development of web and mobile applications for chemical toxicity prediction. J. Braz. Chem. Soc. 29, 982-988. doi: 10.21577/0103-5053.20180013

Andrade, C. H., Neves, B. J., Melo-Filho, C. C., Rodrigues, J., Silva, D. C., Braga, R. C., et al. (2018). In silico chemogenomics drug repositioning strategies for neglected tropical diseases. Curr. Med. Chem. 25, 1-22. doi: 10.2174/0929867325666180309114824

Awale, M., Sirockin, F., Stiefl, N., and Reymond, J.-L. (2019). Drug analogs from fragment-based long short-term memory generative neural networks. J. Chem. Inf. Model. 59, 1347-1356. doi: 10.1021/acs.jcim.8b00902

Barril, X. (2013). Druggability predictions: methods, limitations, and applications. Wiley Interdiscip. Rev. Comput. Mol. Sci. 3, 327-338. doi: 10.1002/wcms.1134

Benmansour, F., Trist, I., Coutard, B., Decroly, E., Querat, G., Brancale, A., et al. (2017). Discovery of novel dengue virus NS5 methyltransferase non-nucleoside inhibitors by fragment-based drug design. Eur. J. Med. Chem. 125, 865-880. doi: 10.1016/j.ejmech.2016.10.007

Berglund, U. W., Sanjiv, K., Gad, H., Kalderén, C., Koolmeister, T., Pham, T., et al. (2016). Validation and development of MTH1 inhibitors for treatment of cancer. Ann. Oncol. 27, 2275-2283. doi: 10.1093/annonc/ mdw429

Boda, K., Seidel, T., and Gasteiger, J. (2007). Structure and reaction based evaluation of synthetic accessibility. J. Comput. Aided. Mol. Des. 21, 311-325. doi: 10.1007/s10822-006-9099-2

Boehr, D. D., Nussinov, R., and Wright, P. E. (2009). The role of dynamic conformational ensembles in biomolecular recognition. Nat. Chem. Biol. 5, 789-796. doi: 10.1038/nchembio.232

Boettcher, A., Ruedisser, S., Erbel, P., Vinzenz, D., Schiering, N., Hassiepen, U., et al. (2010). Fragment-based screening by biochemical assays. J. Biomol. Screen. 15, 1029-1041. doi: 10.1177/1087057110380455

Bohm, H.-J. (1992). The computer program LUDI: a new method for the de novo design of enzyme inhibitors. J. Comput. Aided. Mol. Des. 6, 61-78. doi: $10.1007 /$ BF00124387

Bollag, G., Hirth, P., Tsai, J., Zhang, J., Ibrahim, P. N., Cho, H., et al. (2010). Clinical efficacy of a RAF inhibitor needs broad target blockade in BRAF-mutant melanoma. Nature 467, 596-599. doi: 10.1038/nature09454 contributed to several parts of the manuscript. NF drafted the introduction and one of the case studies. LS drafted the fragment optimization section and one of the case studies. JM-F drafted most of the in silico strategies section and one of the case studies. $\mathrm{BN}$ designed all figures and revised the manuscript. AG and RM drafted the de novo design section and one of the case studies.

\section{ACKNOWLEDGMENTS}

The authors would like to thank Brazilian funding agencies CNPq, CAPES, FAPERJ, FAPEG, and FIOCRUZ for financial support and fellowships. This study was also supported by funding from the United Kingdom's Academy of Medical Sciences Newton Fund (NAF $\backslash$ R2 $\backslash 180641)$.

Bollag, G., Tsai, J., Zhang, J., Zhang, C., Ibrahim, P., Nolop, K., et al. (2012). Vemurafenib: the first drug approved for BRAF-mutant cancer. Nat. Rev. Drug Discov. 11, 873-886. doi: 10.1038/nrd3847

Braga, R., Alves, V., Silva, A., Nascimento, M., Silva, F., Liao, L., et al. (2014). Virtual screening strategies in medicinal chemistry: the state of the art and current challenges. Curr. Top. Med. Chem. 14, 1899-1912. doi: 10.2174/1568026614666140929120749

Braga, R. C., Alves, V. M., Silva, M. F. B., Muratov, E., Fourches, D., Lião, L. M., et al. (2015). Pred-hERG: a novel web-accessible computational tool for predicting cardiac toxicity. Mol. Inform. 34, 698-701. doi: 10.1002/minf.201500040

Chen, Y., and Shoichet, B. K. (2009). Molecular docking and ligand specificity in fragment-based inhibitor discovery. Nat. Chem. Biol. 5, 358-364. doi: $10.1038 /$ nchembio. 155

Cheng, F., Li, W., Zhou, Y., Shen, J., Wu, Z., Liu, G., et al. (2012). admetSAR: a comprehensive source and free tool for assessment of chemical ADMET properties. J. Chem. Inf. Model. 52, 3099-3105. doi: 10.1021/ ci300367a

Cherkasov, A., Muratov, E. N., Fourches, D., Varnek, A., Baskin, I. I., Cronin, M., et al. (2014). QSAR modeling: where have you been? Where are you going to? J. Med. Chem. 57, 4977-5010. doi: 10.1021/jm4004285

Chevillard, F., Rimmer, H., Betti, C., Pardon, E., Ballet, S., van Hilten, N., et al. (2018). Binding-site compatible fragment growing applied to the design of $\beta 2$-adrenergic receptor ligands. J. Med. Chem. 61, 1118-1129. doi: 10.1021/acs.jmedchem.7b01558

Chung, S., Parker, J. B., Bianchet, M., Amzel, L. M., and Stivers, J. T. (2009). Impact of linker strain and flexibility in the design of a fragment-based inhibitor. Nat. Chem. Biol. 5, 407-413. doi: 10.1038/nchembio.163

Ciulli, A. (2013a). Biophysical screening for the discovery of small-molecule ligands. Methods Mol. Biol. 1008, 357-388. doi: 10.1007/978-1-62703-398-5_13

Ciulli, A. (2013b). "Biophysical screening for the discovery of small-molecule ligands," in Methods in Molecular Biology, eds M. A. Williams and T. Daviter (Totowa, NJ: Humana Press), 357-388.

Coutard, B., Decroly, E., Li, C., Sharff, A., Lescar, J., Bricogne, G., et al. (2014). Assessment of Dengue virus helicase and methyltransferase as targets for fragment-based drug discovery. Antiviral Res. 106, 61-70. doi: 10.1016/j.antiviral.2014.03.013

Cox, O. B., Krojer, T., Collins, P., Monteiro, O., Talon, R., Bradley, A., et al. (2016). A poised fragment library enables rapid synthetic expansion yielding the first reported inhibitors of PHIP(2), an atypical bromodomain. Chem. Sci. 7, 2322-2330. doi: 10.1039/C5SC03115J

Cukuroglu, E., Engin, H. B., Gursoy, A., and Keskin, O. (2014). Hot spots in protein-protein interfaces: towards drug discovery. Prog. Biophys. Mol. Biol. 116, 165-173. doi: 10.1016/j.pbiomolbio.2014. 06.003

Daina, A., Michielin, O., and Zoete, V. (2017). SwissADME: a free web tool to evaluate pharmacokinetics, drug-likeness and medicinal chemistry friendliness of small molecules. Sci. Rep. 7:42717. doi: 10.1038/srep42717 
Davis, B. J., and Roughley, S. D. (2017). "Fragment-based lead discovery," in Annual Reports in Medicinal Chemistry, ed R. A. Goodnow (London: Elsevier Inc.), 371-439. doi: 10.1016/bs.armc.2017.07.002

De Fusco, C., Brear, P., Iegre, J., Georgiou, K. H., Sore, H. F., Hyvönen, M., et al. (2017). A fragment-based approach leading to the discovery of a novel binding site and the selective CK2 inhibitor CAM4066. Bioorg. Med. Chem. 25, 3471-3482. doi: 10.1016/j.bmc.2017.04.037

Dey, F., and Caflisch, A. (2008). Fragment-based de novo ligand design by multiobjective evolutionary optimization. J. Chem. Inf. Model. 48, 679-690. doi: $10.1021 / \mathrm{ci} 700424 \mathrm{~b}$

Donald, A., McHardy, T., Rowlands, M. G., Hunter, L.-J. K., Davies, T. G., Berdini, V., et al. (2007). Rapid evolution of 6-phenylpurine inhibitors of protein kinase B through structure-based design. J. Med. Chem. 50, 2289-2292. doi: $10.1021 /$ jm0700924

Dong, H., Zhang, B., and Shi, P.-Y. (2008). Flavivirus methyltransferase: a novel antiviral target. Antiviral Res. 80, 1-10. doi: 10.1016/j.antiviral.2008.05.003

Dong, J., Wang, N.-N., Yao, Z.-J., Zhang, L., Cheng, Y., Ouyang, D., et al. (2018). ADMETlab: a platform for systematic ADMET evaluation based on a comprehensively collected ADMET database. J. Cheminform. 10:29. doi: 10.1186/s13321-018-0283-x

Durrant, J. D., Amaro, R. E., and McCammon, J. A. (2009). AutoGrow: a novel algorithm for protein inhibitor design. Chem. Biol. Drug Des. 73, 168-178. doi: 10.1111/j.1747-0285.2008.00761.x

Durrant, J. D., Lindert, S., and McCammon, J. A. (2013). AutoGrow 3.0: an improved algorithm for chemically tractable, semi-automated protein inhibitor design. J. Mol. Graph. Model. 44, 104-112. doi: 10.1016/j.jmgm.2013.05.006

Erlanson, D. A., Davis, B. J., and Jahnke, W. (2019). Fragment-based drug discovery: advancing fragments in the absence of crystal structures. Cell Chem. Biol. 26, 9-15. doi: 10.1016/j.chembiol.2018.10.001

Erlanson, D. A., Fesik, S. W., Hubbard, R. E., Jahnke, W., and Jhoti, H. (2016). Twenty years on: the impact of fragments on drug discovery. Nat. Rev. Drug Discov. 15, 605-619. doi: 10.1038/nrd.2016.109

Ertl, P., and Schuffenhauer, A. (2009). Estimation of synthetic accessibility score of drug-like molecules based on molecular complexity and fragment contributions. J. Cheminform. 1:8. doi: 10.1186/1758-2946-1-8

Ferreira, L. L. G., and Andricopulo, A. D. (2019). ADMET modeling approaches in drug discovery. Drug Discov. Today 24, 1157-1165. doi: 10.1016/j.drudis.2019.03.015

Fukunishi, Y., Kurosawa, T., Mikami, Y., and Nakamura, H. (2014). Prediction of synthetic accessibility based on commercially available compound databases. J. Chem. Inf. Model. 54, 3259-3267. doi: 10.1021/ci500568d

Gawehn, E., Hiss, J. A., Brown, J. B., and Schneider, G. (2018). Advancing drug discovery via GPU-based deep learning. Expert Opin. Drug Discov. 13, 579-582. doi: 10.1080/17460441.2018.1465407

Giordanetto, F., Jin, C., Willmore, L., Feher, M., and Shaw, D. E. (2019). Fragment hits: what do they look like and how do they bind? J. Med. Chem. 62, 3381-3394. doi: 10.1021/acs.jmedchem.8b01855

Godemann, R., Madden, J., Krämer, J., Smith, M., Fritz, U., Hesterkamp, T., et al. (2009). Fragment-based discovery of BACE1 inhibitors using functional assays. Biochemistry 48, 10743-10751. doi: 10.1021/bi901061a

Gómez-Bombarelli, R., Wei, J. N., Duvenaud, D., Hernández-Lobato, J. M., Sánchez-Lengeling, B., Sheberla, D., et al. (2018). Automatic chemical design using a data-driven continuous representation of molecules. ACS Cent. Sci. 4, 268-276. doi: 10.1021/acscentsci.7b00572

Grove, L. E., Vajda, S., and Kozakov, D. (2016). "Computational methods to support fragment-based drug discovery," in Fragment-Based Drug Discovery: Lessons and Outlook, eds D. A. Erlanson and W. Jahnke (Weinheim: WileyVCH Verlag GmbH and Co. KGaA), 197-222.

Gupta, A., Müller, A. T., Huisman, B. J. H., Fuchs, J. A., Schneider, P., and Schneider, G. (2018). Generative recurrent networks for de novo drug design. Mol. Inform. 37:1700111. doi: 10.1002/minf.2017 00111

Hall, D. R., Ngan, C. H., Zerbe, B. S., Kozakov, D., and Vajda, S. (2012). Hot spot analysis for driving the development of hits into leads in fragment-based drug discovery. J. Chem. Inf. Model. 52, 199-209. doi: 10.1021/ci200468p

Hall, R. J., Mortenson, P. N., and Murray, C. W. (2014). Efficient exploration of chemical space by fragment-based screening. Prog. Biophys. Mol. Biol. 116, 82-91. doi: 10.1016/j.pbiomolbio.2014.09.007
Hall, R. J., Murray, C. W., and Verdonk, M. L. (2017). The fragment network: a chemistry recommendation engine built using a graph database. J. Med. Chem. 60, 6440-6450. doi: 10.1021/acs.jmedchem.7b00809

Hann, M. M., Leach, A. R., and Harper, G. (2001). Molecular complexity and its impact on the probability of finding leads for drug discovery. J. Chem. Inf. Comput. Sci. 41, 856-864. doi: 10.1021/ci000403i

Harel, S., and Radinsky, K. (2018). Prototype-based compound discovery using deep generative models. Mol. Pharm. 15, 4406-4416. doi: 10.1021/acs.molpharmaceut.8b00474

Henzler-Wildman, K., and Kern, D. (2007). Dynamic personalities of proteins. Nature 450, 964-972. doi: 10.1038/nature06522

Hoffer, L., Voitovich, Y. V., Raux, B., Carrasco, K., Muller, C., Fedorov, A. Y., et al. (2018). Integrated strategy for lead optimization based on fragment growing: the diversity-oriented-target-focused-synthesis approach. J. Med. Chem. 61, 5719-5732. doi: 10.1021/acs.jmedchem.8b00653

Hopkins, A. L., Groom, C. R., and Alex, A. (2004). Ligand efficiency: a useful metric for lead selection. Drug Discov. Today 9, 430-431. doi: 10.1016/S1359-6446(04)03069-7

Hubbard, R. (2015). Current perspectives in fragment-based ligand discovery. Acta Crystallogr. Sect. A Found. Adv. 71:s37. doi: 10.1107/S2053273315099416

Hubbard, R. E., and Murray, J. B. (2011). Experiences in Fragment-Based Lead Discovery, 1st Edn. Oxford, UK: Elsevier Inc.

Hung, A. W., Silvestre, H. L., Wen, S., Ciulli, A., Blundell, T. L., and Abell, C. (2009). Application of fragment growing and fragment linking to the discovery of inhibitors of Mycobacterium tuberculosis pantothenate synthetase. Angew. Chemie Int. Ed. 48, 8452-8456. doi: 10.1002/anie.200903821

Hussein, H. A., Borrel, A., Geneix, C., Petitjean, M., Regad, L., and Camproux, A.-C. (2015). PockDrug-server: a new web server for predicting pocket druggability on holo and apo proteins. Nucleic Acids Res. 43, W436-W442. doi: 10.1093/nar/gkv462

Ichihara, O., Barker, J., Law, R. J., and Whittaker, M. (2011). Compound design by fragment-linking. Mol. Inform. 30, 298-306. doi: 10.1002/minf.201000174

Jacquemard, C., Drwal, M. N., Desaphy, J., and Kellenberger, E. (2019). Binding mode information improves fragment docking. J Cheminform 11:24. doi: 10.1186/s13321-019-0346-7

Joseph-mccarthy, D., Campbell, A. J., Kern, G., and Moustakas, D. (2013). Fragment-based lead discovery and design. J. Chem. Inf. Model. 54, 693-704. doi: 10.1021/ci400731w

Kozakov, D., Grove, L. E., Hall, D. R., Bohnuud, T., Mottarella, S. E., Luo, L., et al. (2015). The FTMap family of web servers for determining and characterizing ligand-binding hot spots of proteins. Nat Protoc 10, 733-755. doi: 10.1038/nprot.2015.043

Kumar, A., Voet, A., and Zhang, K. Y. J. (2012). Fragment based drug design: from experimental to computational approaches. Curr. Med. Chem. 19, 5128-5147. doi: 10.2174/092986712803530467

Lamoree, B., and Hubbard, R. E. (2017). Current perspectives in fragment-based lead discovery (FBLD). Essays Biochem. 61, 453-464. doi: 10.1042/EBC20170028

Li, Y., Zhang, L., and Liu, Z. (2018). Multi-objective de novo drug design with conditional graph generative model. J. Cheminform. 10, 1-24. doi: 10.1186/s13321-018-0287-6

Lin, F.-Y., Esposito, E. X., and Tseng, Y. J. (2018). LeadOp+R: structurebased lead optimization with synthetic accessibility. Front. Pharmacol. 9:96. doi: 10.3389/fphar.2018.00096

Lo, M.-C., Aulabaugh, A., Jin, G., Cowling, R., Bard, J., Malamas, M., et al. (2004). Evaluation of fluorescence-based thermal shift assays for hit identification in drug discovery. Anal. Biochem. 332, 153-159. doi: 10.1016/j.ab.2004. 04.031

Macarron, R., Banks, M. N., Bojanic, D., Burns, D. J., Cirovic, D. A., Garyantes, T., et al. (2011). Impact of high-throughput screening in biomedical research. Nat. Rev. Drug Discov. 10, 188-195. doi: 10.1038/nrd3368

Mak, K. K., and Pichika, M. R. (2019). Artificial intelligence in drug development: present status and future prospects. Drug Discov. Today 24, 773-780. doi: 10.1016/j.drudis.2018.11.014

Männel, B., Jaiteh, M., Zeifman, A., Randakova, A., Möller, D., Hübner, H., et al. (2017). Structure-guided screening for functionally selective D 2 dopamine receptor ligands from a virtual chemical library. ACS Chem. Biol. 12, 2652-2661. doi: 10.1021/acschembio.7b00493 
Matias, P. M., Donner, P., Coelho, R., Thomaz, M., Peixoto, C., Macedo, S., et al. (2000). Structural evidence for ligand specificity in the binding domain of the human androgen receptor: implications for pathogenic gene mutations. J. Biol. Chem. 275, 26164-26171. doi: 10.1074/jbc.M004571200

Merk, D., Friedrich, L., Grisoni, F., and Schneider, G. (2018). De novo design of bioactive small molecules by artificial intelligence. Mol. Inform. 37, 3-6. doi: 10.1002/minf.201700153

Mitcheson, J. S., Chen, J., Lin, M., Culberson, C., and Sanguinetti, M. C. (2000). A structural basis for drug-induced long QT syndrome. Proc. Natl. Acad. Sci. U.S.A. 97, 12329-12333. doi: 10.1073/pnas.210244497

Miyake, Y., Itoh, Y., Hatanaka, A., Suzuma, Y., Suzuki, M., Kodama, H., et al. (2019). Identification of novel lysine demethylase 5-selective inhibitors by inhibitor-based fragment merging strategy. Bioorg. Med. Chem. 27, 1119-1129. doi: 10.1016/j.bmc.2019.02.006

Mondal, M., Groothuis, D. E., and Hirsch, A. K. H. (2015). Fragment growing exploiting dynamic combinatorial chemistry of inhibitors of the aspartic protease endothiapepsin. Medchemcomm 6, 1267-1271. doi: 10.1039/C5MD00157A

Navratilova, I., and Hopkins, A. L. (2010). Fragment screening by surface plasmon resonance. ACS Med. Chem. Lett. 1, 44-48. doi: 10.1021/ml900002k

Neves, B. J., Braga, R. C., Melo-Filho, C. C., Moreira-Filho, J. T., Muratov, E. N., and Andrade, C. H. (2018). QSAR-based virtual screening: advances and applications in drug discovery. Front. Pharmacol. 9:1275. doi: $10.3389 /$ fphar.2018.01275

Ngan, C. H., Bohnuud, T., Mottarella, S. E., Beglov, D., Villar, E. A., Hall, D. R., et al. (2012). FTMAP: extended protein mapping with user-selected probe molecules. Nucleic Acids Res. 40, W271-W275. doi: 10.1093/nar/gks441

Nikiforov, P. O., Surade, S., Blaszczyk, M., Delorme, V., Brodin, P., Baulard, A. R., et al. (2016). A fragment merging approach towards the development of small molecule inhibitors of Mycobacterium tuberculosis EthR for use as ethionamide boosters. Org. Biomol. Chem. 14, 2318-2326. doi: 10.1039/C5OB02630J

Nissink, J. W. M. (2009). Simple size-independent measure of ligand efficiency. J. Chem. Inf. Model. 49, 1617-1622. doi: 10.1021/ci900094m

Olivecrona, M., Blaschke, T., Engkvist, O., and Chen, H. (2017). Molecular denovo design through deep reinforcement learning. J. Cheminform. 9, 1-14. doi: 10.1186/s13321-017-0235-x

Pascoini, A. L., Federico, L. B., Arêas, A. L. F., Verde, B. A., Freitas, P. G., and Camps, I. (2019). In silico development of new acetylcholinesterase inhibitors. J. Biomol. Struct. Dyn. 37, 1007-1021. doi: 10.1080/07391102.2018. 1447513

Patel, D., Bauman, J. D., and Arnold, E. (2014). Advantages of crystallographic fragment screening: functional and mechanistic insights from a powerful platform for efficient drug discovery. Prog. Biophys. Mol. Biol. 116, 92-100. doi: 10.1016/j.pbiomolbio.2014.08.004

Pedro, L., and Quinn, R. (2016). Native mass spectrometry in fragment-based drug discovery. Molecules 21:E984. doi: 10.3390/molecules21080984

Pegg, S. C. H., Haresco, J. J., and Kuntz, I. D. (2001). A genetic algorithm for structure-based de novo design. J. Comput. Aided. Mol. Des. 15, 911-933. doi: 10.1023/A:1014389729000

Pérez-Sianes, J., Pérez-Sánchez, H., and Díaz, F. (2018). Virtual screening meets deep learning. Curr. Comput. Aided. Drug Des. 15, 6-28. doi: $10.2174 / 1573409914666181018141602$

Pierce, A. C., Rao, G., and Bemis, G. W. (2004). BREED: generating novel inhibitors through hybridization of known ligands. Application to CDK2, P38, and HIV protease. J. Med. Chem. 47, 2768-2775. doi: 10.1021/jm030543u

Podolyan, Y., Walters, M. A., and Karypis, G. (2010). Assessing synthetic accessibility of chemical compounds using machine learning methods. J. Chem. Inf. Model. 50, 979-991. doi: 10.1021/ci900301v

Polinsky, R. J. (1998). Clinical pharmacology of rivastigmine: a new-generation acetylcholinesterase inhibitor for the treatment of alzheimer's disease. Clin. Ther. 20, 634-647. doi: 10.1016/S0149-2918(98)80127-6

Polykovskiy, D., Zhebrak, A., Vetrov, D., Ivanenkov, Y., Aladinskiy, V., Mamoshina, P., et al. (2018). Entangled conditional adversarial autoencoder for de novo drug discovery. Mol. Pharm. 15, 4398-4405. doi: 10.1021/acs.molpharmaceut.8b00839

Popova, M., Isayev, O., and Tropsha, A. (2018). Deep reinforcement learning for de novo drug design. Sci. Adv. 4:eaap7885. doi: 10.1126/sciadv.aap7885
Putin, E., Asadulaev, A., Ivanenkov, Y., Aladinskiy, V., Sanchez-Lengeling, B., Aspuru-Guzik, A., et al. (2018). Reinforced adversarial neural computer for de novo molecular design. J. Chem. Inf. Model. 58, 1194-1204. doi: 10.1021/acs.jcim.7b00690

Ramsundar, B., Liu, B., Wu, Z., Verras, A., Tudor, M., Sheridan, R. P., et al. (2017). Is multitask deep learning practical for pharma? J. Chem. Inf. Model. 57, 2068-2076. doi: 10.1021/acs.jcim.7b00146

Riniker, S., and Landrum, G. A. (2013). Open-source platform to benchmark fingerprints for ligand-based virtual screening. Riniker Landrum J. Cheminformatics 5:26. doi: 10.1186/1758-2946-5-26

Rodríguez, D., Chakraborty, S., Warnick, E., Crane, S., Gao, Z.-G., O’Connor, R., et al. (2016). Structure-based screening of uncharted chemical space for atypical adenosine receptor agonists. ACS Chem. Biol. 11, 2763-2772. doi: $10.1021 /$ acschembio.6b00357

Rogers, D., and Hahn, M. (2010). Extended-connectivity fingerprints. J. Chem. Inf. Model. 50, 742-754. doi: 10.1021/ci100050t

Rudling, A., Gustafsson, R., Almlöf, I., Homan, E., Scobie, M., Warpman Berglund, U., et al. (2017). Fragment-based discovery and optimization of enzyme inhibitors by docking of commercial chemical space. J. Med. Chem. 60, 8160-8169. doi: 10.1021/acs.jmedchem.7b01006

Ryan, A., Polycarpou, E., Lack, N. A., Evangelopoulos, D., Sieg, C., Halman, A., et al. (2017). Investigation of the mycobacterial enzyme $\mathrm{HsaD}$ as a potential novel target for anti-tubercular agents using a fragment-based drug design approach. Br. J. Pharmacol. 174, 2209-2224. doi: 10.1111/bph. 13810

Schmidtke, P., and Barril, X. (2010). Understanding and predicting druggability. A high-throughput method for detection of drug binding sites. J. Med. Chem. 53, 5858-5867. doi: 10.1021/jm100574m

Schneider, G. (2018). Generative models for artificially-intelligent molecular design. Mol. Inform. 37:1880131. doi: 10.1002/minf.201880131

Schneider, G., and Clark, D. E. (2019). Automated de novo drug design: are we nearly there yet? Angew. Chemie Int. Ed. 58, 10792-10803. doi: 10.1002/anie.201814681

Schneider, G., and Fechner, U. (2005). Computer-based de novo design of drug-like molecules. Nat. Rev. Drug Discov. 4, 649-663. doi: 10.1038/nrd1799

Schuffenhauer, A., Ruedisser, S., Marzinzik, A., Jahnke, W., Selzer, P., and Jacoby, E. (2005). Library design for fragment based screening. Curr. Top. Med. Chem. 5, 751-762. doi: 10.2174/1568026054637700

Schulz, M. N., Landström, J., Bright, K., and Hubbard, R. E. (2011). Design of a fragment library that maximally represents available chemical space. J. Comput. Aided. Mol. Des. 25, 611-620. doi: 10.1007/s10822-011-9461-x

Schyman, P., Liu, R., Desai, V., and Wallqvist, A. (2017). vNN web server for ADMET predictions. Front. Pharmacol. 8:889. doi: 10.3389/fphar.2017.00889

Segler, M. H. S., Kogej, T., Tyrchan, C., and Waller, M. P. (2018). Generating focused molecule libraries for drug discovery with recurrent neural networks. ACS Cent. Sci. 4, 120-131. doi: 10.1021/acscentsci.7b00512

Seo, M.-H., Park, J., Kim, E., Hohng, S., and Kim, H.-S. (2014). Protein conformational dynamics dictate the binding affinity for a ligand. Nat. Commun. 5:3724. doi: 10.1038/ncomms4724

Shuker, S. B., Hajduk, P. J., Meadows, R. P., and Fesik, S. W. (1996). Discovering high-affinity ligands for proteins: SAR by NMR. Science 274, 1531-1534. doi: $10.1126 /$ science. 274.5292 .1531

Shultz, M. D. (2013). Setting expectations in molecular optimizations: strengths and limitations of commonly used composite parameters. Bioorg. Med. Chem. Lett. 23, 5980-5991. doi: 10.1016/j.bmcl.2013.08.029

Silvestre, H. L., Blundell, T. L., Abell, C., and Ciulli, A. (2013). Integrated biophysical approach to fragment screening and validation for fragmentbased lead discovery. Proc. Natl. Acad. Sci. U.S.A. 110, 12984-12989. doi: 10.1073/pnas. 1304045110

Smits, V. A. J., and Gillespie, D. A. (2014). Cancer therapy: targeting the poison within. Cell Cycle 13, 2330-2333. doi: 10.4161/cc.29756

Srinivas Reddy, A., Chen, L., and Zhang, S. (2013). "Structure-based de novo drug design," in De novo Molecular Design, ed. G. Schneider (Weinheim: Wiley-VCH Verlag GmbH and Co. KGaA), 97-124.

Sterling, T., and Irwin, J. J. (2015). ZINC 15 - ligand discovery for everyone. J. Chem. Inf. Model. 55, 2324-2337. doi: 10.1021/acs.jcim.5b00559

Strecker, C., Peters, H., Hackl, T., Peters, T., and Meyer, B. (2019). Fragment growing to design optimized inhibitors for human blood 
group B Galactosyltransferase (GTB). ChemMedChem 1, 1336-1342. doi: $10.1002 / \mathrm{cmdc} .201900296$

Talesa, V. N. (2001). Acetylcholinesterase in Alzheimer's disease. Mech. Ageing Dev. 122, 1961-1969. doi: 10.1016/S0047-6374(01)00309-8

Thomas, S. E., Mendes, V., Kim, S. Y., Malhotra, S., Ochoa-Montaño, B., Blaszczyk, M., et al. (2017). Structural biology and the design of new therapeutics: from HIV and cancer to mycobacterial infections. J. Mol. Biol. 429, 2677-2693. doi: 10.1016/j.jmb.2017.06.014

Torres, P. H. M., Sodero, A. C. R., Jofily, P., and Silva, F. P. Jr. (2019). Key topics in molecular docking for drug design. Int. J. Mol. Sci. 20:4574. doi: 10.3390/ijms20184574

Trevizani, R., Custódio, F. L., dos Santos, K. B., and Dardenne, L. E. (2017). Critical features of fragment libraries for protein structure prediction. PLoS ONE 12:e0170131. doi: 10.1371/journal.pone.0170131

Tropsha, A. (2010). Best practices for QSAR model development, validation, and exploitation. Mol. Inform. 29, 476-488. doi: 10.1002/minf.201000061

Tversky, A. (1977). Features of similarity. Psychol. Rev. 84, 327-352. doi: 10.1037/0033-295X.84.4.327

Vamathevan, J., Clark, D., Czodrowski, P., Dunham, I., Ferran, E., Lee, G., et al. (2019). Applications of machine learning in drug discovery and development. Nat. Rev. Drug Discov. 18, 463-477. doi: 10.1038/s41573-019-0024-5

Veber, D. F., Johnson, S. R., Cheng, H.-Y., Smith, B. R., Ward, K. W., and Kopple, K. D. (2002). Molecular properties that influence the oral bioavailability of drug candidates. J. Med. Chem. 45, 2615-2623. doi: 10.1021/jm020017n

Verdonk, M. L., Giangreco, I., Hall, R. J., Korb, O., Mortenson, P. N., and Murray, C. W. (2011). Docking performance of fragments and druglike compounds. J. Med. Chem. 54, 5422-5431. doi: 10.1021/jm200558u

Verdonk, M. L., and Hartshorn, M. J. (2004). Structure-guided fragment screening for lead discovery. Curr. Opin. Drug Discov. Devel. 7, 404-410.

Wang, R., Gao, Y., and Lai, L. (2000). LigBuilder: a multi-purpose program for structure-based drug design. J. Mol. Model. 6, 498-516. doi: $10.1007 / \mathrm{s} 0089400060498$

Wang, T., Wu, M.-B., Chen, Z.-J., Chen, H., Lin, J.-P., and Yang, L.-R. (2015). Fragment-based drug discovery and molecular docking in drug design. Curr. Pharm. Biotechnol. 16, 11-25. doi: 10.2174/1389201015666141122 204532

Whitehouse, A. J., Thomas, S. E., Brown, K. P., Fanourakis, A., Chan, D. S.-H., Libardo, M. D. J., et al. (2019). Development of inhibitors against Mycobacterium abscessus tRNA (m 1 G37) methyltransferase (TrmD) using fragment-based approaches. J. Med. Chem. 62, 7210-7232. doi: 10.1021/acs.jmedchem.9b00809

Willighagen, E. L., Waagmeester, A., Spjuth, O., Ansell, P., Williams, A. J., Tkachenko, V., et al. (2013). The ChEMBL database as linked open data. J. Cheminform. 5:23. doi: 10.1186/1758-2946-5-23

Xu, X., Fang, X., Wang, J., and Zhu, H. (2017). Identification of novel ROS inducer by merging the fragments of piperlongumine and dicoumarol. Bioorg. Med. Chem. Lett. 27, 1325-1328. doi: 10.1016/j.bmcl.2016.08.016

Yang, H., Lou, C., Sun, L., Li, J., Cai, Y., Wang, Z., et al. (2019a). admetSAR 2.0: web-service for prediction and optimization of chemical ADMET properties. Bioinformatics 35, 1067-1069. doi: 10.1093/bioinformatics/ bty707

Yang, X., Wang, Y., Byrne, R., Schneider, G., and Yang, S. (2019b). Concepts of artificial intelligence for computer-assisted drug discovery. Chem. Rev. 119, 10520-10594. doi: 10.1021/acs.chemrev.8b00728

Yuan, Y., Pei, J., and Lai, L. (2011). LigBuilder 2: a practical de novo drug design approach. J. Chem. Inf. Model. 51, 1083-1091. doi: 10.1021/ci100350u

Conflict of Interest: The authors declare that the research was conducted in the absence of any commercial or financial relationships that could be construed as a potential conflict of interest.

Copyright (C) 2020 de Souza Neto, Moreira-Filho, Neves, Maidana, Guimarães, Furnham, Andrade and Silva. This is an open-access article distributed under the terms of the Creative Commons Attribution License (CC BY). The use, distribution or reproduction in other forums is permitted, provided the original author(s) and the copyright owner(s) are credited and that the original publication in this journal is cited, in accordance with accepted academic practice. No use, distribution or reproduction is permitted which does not comply with these terms. 